\title{
Study on the Antihypertensive Mechanism of Astragalus membranaceus and Salvia miltiorrhiza Based on Intestinal Flora-Host Metabolism
}

\author{
Cong Han $\mathbb{D},{ }^{1}$ Yue-hua Jiang $\mathbb{D},{ }^{2}$ Wei Li $\mathbb{D},{ }^{3}$ Yao Liu $\mathbb{D},{ }^{1}$ and Zhen-qiang Qi $\mathbb{D}{ }^{1}$ \\ ${ }^{1}$ Shandong University of Traditional Chinese Medicine, Jinan 250014, China \\ ${ }^{2}$ Central Laboratory of Affiliated Hospital of Shandong University of Traditional Chinese Medicine, Jinan 250014, China \\ ${ }^{3}$ Nephropathy Department, Affiliated Hospital of Shandong University of Traditional Chinese Medicine, Jinan 250014, China
}

Correspondence should be addressed to Wei Li; lweidw@163.com

Received 17 March 2019; Accepted 26 June 2019; Published 21 July 2019

Academic Editor: Nunziatina De Tommasi

Copyright (C) 2019 Cong Han et al. This is an open access article distributed under the Creative Commons Attribution License, which permits unrestricted use, distribution, and reproduction in any medium, provided the original work is properly cited.

Our previous studies have shown that the combination of Astragalus membranaceus and Salvia miltiorrhiza (HD) had a good antihypertensive effect, but its potential mechanism remained unclear. This study aimed to investigate the role of intestinal flora and serum metabolism induced by HD against hypertension. 16 spontaneous hypertensive rats (SHRs) were divided into HD group $(5.9 \mathrm{~g} / \mathrm{kg}$ ) and model group (M) (normal saline), with eight Wistar-Kyoto (WKY) rats as control group (W) (normal saline). Rats were fed by gavage once a day for 28 days. The changes of intestinal flora and serum metabolism were analyzed by $16 \mathrm{~S}$ rDNA sequencing and LC-MS/MS assay. HD decreased blood pressure steadily, improved the structure and composition of imbalance flora in SHRs, increased the abundance and diversity of flora, and decreased flora Firmicutes to Bacteroidetes (F/B) ratio. Rumen bacterium NK4A214, Clostridium sp. MC 40 increased remarkably in M group. Akkermansia, Akkermansia muciniphila, and Lactobacillus intestinalis increased significantly in HD group, which were functionally related to the significant increase of Lachnoclostridium, Faecalibaculum, and Lactobacillus reuteri in W group, which were all probiotics producing butyric acid, lactic acid, and regulating inflammation and other antihypertensive related factors. HD also changed the serum metabolic pattern of SHRs. 16 potential biomarkers related to inflammation, vasodilation, steroid hormones, oxidative stress, and etc. changed significantly, mainly enriched in arachidonic acid metabolism, tryptophan metabolism, steroid hormone biosynthesis, and glutathione metabolism. The correlation analysis demonstrated that the dominant genius and species in three groups were highly correlated with steroid hormone biosynthesis, arachidonic acid metabolism, tryptophan metabolism, and vitamin B6 metabolism. Our research indicated that HD had a good antihypertensive effect, which may be driven by the protective intestinal flora and beneficial metabolites induced by it, and the metabolites were closely related to the changes of intestinal flora. It provided new insights for the antihypertensive mechanism of HD.

\section{Introduction}

The intestinal flora as the second genome of the human body was not only directly involved in the normal metabolism but also closely related to the occurrence and development of hypertension [1-6]. It had been found that both spontaneous hypertensive rats (SHRs) and hypertensive patients had the microecological imbalance of the richness, diversity, and uniformity in intestinal flora and the increased ratio of Firmicutes to Bacteroidetes [4]. In addition, a meta-analysis of multiple randomized human clinical trials had shown that probiotics could effectively lower blood pressure [5]. A recent study found that blood pressure was elevated by fecal transplantation from hypertensive human donors to germ-free mice. And their study also confirmed that serum metabolic changes in patients with prehypertension and hypertension were closely related to intestinal flora imbalance [6]. Metabolomics, as a powerful tool for identifying the changes of endogenous metabolites in pharmacodynamics, had been widely used in exploring the pathological mechanism of cardiovascular and cerebrovascular diseases such as hypertension in recent years [7]. The metabolic studies 
of SHRs had found that the changes of metabolites in the pathways of arachidonic acid metabolism, lipid metabolism, fatty acid metabolism, vitamin metabolism, and amino acid metabolism were closely related to the regulation of blood pressure [8-10]. However, the specific mechanism between intestinal flora-host metabolism and blood pressure regulation has not been fully elucidated.

Astragalus membranaceus (of the Leguminosae family), with Chinese name of Huang qi and the Latin name of Astragalus membranaceus (Fisch.) Bge., is well used in Traditional Chinese Medicines (TCM) for the treatment of general weakness to increase overall immunity. Salvia miltiorrhiza (of the Lamiaceae family), with Chinese name of Dan shen and the Latin name of Salvia miltiorrhiza Bge., is highly valued in TCM for activating blood circulation to dissipate blood stasis. Both of them have been widely used in the treatment of hypertension in China. It had been found that astragaloside IV, an effective component of Astragalus membranaceus, reduced blood pressure in rats with metabolic syndrome by regulating lipid metabolism and endotheliumdependent vasodilation [11]. Salvia miltiorrhiza aqueous extract reduced blood pressure by inhibiting SHRs' vascular remodeling and oxidative stress [12]. The combination of Astragalus membranaceus and Salvia miltiorrhiza (HD) could reduce blood lipid and improve insulin resistance, antiinflammation, and anticoagulation [13-15], all of which were risk factors of hypertension. Our previous studies had found that the combination of HD had better antihypertensive effect than the separated use of herb, but the pharmacological mechanism of blood pressure reduction remained to be further clarified.

Therefore, we speculate that the combination of HD may be involved in the regulation of blood pressure by inducing changes in intestinal flora and serum metabolites. In the present study, we performed $16 \mathrm{~S}$ rDNA V4 region high throughput sequencing on intestinal flora and LC-MS/MS assay on serum metabolites and analyzed the correlation between intestinal flora and serum metabolism, to explore the potential antihypertensive mechanism of combination of HD.

\section{Materials and Methods}

2.1. Preparation of HD. HD were purchased from the Affiliated Hospital of Shandong University of Traditional Chinese Medicine (Jinan, China) and authenticated by Professor Chuanjiang Ma (Chief Pharmacist of Affiliated Hospital of Shandong University of Traditional Chinese Medicine). According to our previous studies and other reports [16], $\mathrm{HD}$ were prepared in a ratio of 2:1, adding 10-fold volume of water to the medicinal material, decocted twice, mixed, and concentrated to $0.59 \mathrm{~g}$ of crude drug per $\mathrm{ml}$.

2.2. Animal and Drug Administration. The research protocol was approved by the Animal Experimental Ethics Committee of the Affiliated Hospital of Shandong University of Traditional Chinese Medicine. Sixteen 16-week-old male SHRs were divided into two groups: HD group (HD) and model group $(\mathrm{M})(\mathrm{n}=8)$ and eight male Wistar-Kyoto (WKY) rats of the same age as control group (W), all of which were purchased from Vital River Laboratory Animal Technology Co. Ltd. (Beijing, China) with animal license number SCXK (Beijing) 2016-0006. The HD group was intragastrically administrated with $\mathrm{HD}(0.59 \mathrm{~g} / \mathrm{mL}, 10 \mathrm{~mL} / \mathrm{kg})$, and rats in $\mathrm{M}$ group and $\mathrm{W}$ group were administrated with the same amount of normal saline. All animals were fed by gavage once a day for 28 days.

2.3. Blood Pressure Measurement. The blood pressure of rats' tail artery was measured by noninvasive blood pressure meter before and 1,2,3, and 4 weeks after administration. The data were expressed by means $\pm \mathrm{SD}$, and multiple comparisons were performed by one-way analysis of variance (ANOVA) using SPSS 19.0 (SPSS Inc., USA).

\subsection{Sample Collection and Preparation}

Serum Sample Preparation. Rats were anesthetized by intraperitoneal injection of $3 \%$ pentobarbital sodium $(40 \mathrm{mg} / \mathrm{kg})$ and serum samples were collected from the abdominal aorta of each rat on the 28th day and immediately centrifuged at 3,000 rpm for $10 \mathrm{~min}$. The supernatant was transferred into a clean tube and stored at $-80^{\circ} \mathrm{C}$. Prior to analysis, $100 \mathrm{uL}$ serum was resuspended with prechilled $80 \%$ methanol $\left(-20^{\circ} \mathrm{C}\right)$ followed by well vortexing. The samples were incubated at $-20^{\circ} \mathrm{C}$ for $60 \mathrm{~min}$ and then were centrifuged at $14000 \mathrm{~g}, 4^{\circ} \mathrm{C}$ for $20 \mathrm{~min}$. The supernatants were spun in a vacuum concentrator until they dry. The dried metabolite pellets were reconstituted by $60 \%$ methanol and analyzed by LC-MS/MS, mixing the same amount of supernatant from each processed sample as a QC sample.

Fecal Collection. Fecal samples were collected directly from the intestines of each sacrificed rat and placed in sterile EP tubes at the end of week 4 and stored at $-80^{\circ} \mathrm{C}$.

HE Staining. All rats were sacrificed on the 28th day and colon tissues were taken and fixed in neutral paraformaldehyde for 48 hours. $4 \mu \mathrm{m}$ sections were cut and embedded in paraffin, stained with hematoxylin-eosin, and sealed with neutral gum for observation by light microscope.

\subsection{Serum UHPLC-MS/MS Analysis}

UHPLC-MS/MS Conditions. LC-MS/MS analyses were performed using a Vanquish UHPLC system (Thermo Fisher, USA) coupled with an Orbitrap Q Exactive HF-X mass spectrometer (Thermo Fisher, USA) operating in the datadependent acquisition (DDA) mode. Samples were injected onto an Accucore HILIC column $(100 \times 2.1 \mathrm{~mm}, 2.6 \mu \mathrm{m})$ using a $16 \mathrm{~min}$ linear gradient at a flow rate of $0.3 \mathrm{~mL} / \mathrm{min}$. The eluents for the positive polarity mode were eluent A $(0.1 \% \mathrm{FA}$ in $95 \% \mathrm{ACN}, 10 \mathrm{mM}$ ammonium acetate) and eluent $\mathrm{B}(0.1 \%$ $\mathrm{FA}$ in $50 \% \mathrm{ACN}, 10 \mathrm{mM}$ ammonium acetate). The eluents for the negative polarity mode were eluent A $(95 \% \mathrm{ACN}$, $10 \mathrm{mM}$ ammonium acetate, $\mathrm{pH} 9.0)$ and eluent $\mathrm{B}$ (50\% ACN, 
$10 \mathrm{mM}$ ammonium acetate, $\mathrm{pH} 9.0$ ). The solvent gradient was set as follows: $2 \% \mathrm{~B}, 1.5 \mathrm{~min} ; 2-100 \% \mathrm{~B}, 12.0 \mathrm{~min} ; 100 \% \mathrm{~B}$, $14.0 \mathrm{~min} ; 100-2 \%$ B, $14.1 \mathrm{~min} ; 2 \%$ B, 16 min. Q Exactive HF-X mass spectrometer was operated in positive/negative polarity mode with spray voltage of $3.2 \mathrm{kV}$, capillary temperature of $320^{\circ} \mathrm{C}$, sheath gas flow rate of $35 \mathrm{arb}$, and aux gas flow rate of 10 arb.

Data Processing and Statistical Analysis. The raw data files generated by UHPLC-MS/MS were processed using the Compound Discoverer 3.0 (CD 3.0, Thermo Fisher, USA) to perform peak alignment, peak picking, and quantitation for each metabolite. After that, peak intensities were normalized to the total spectral intensity. The normalized data was used to predict the molecular formula based on additive ions, molecular ion peaks, and fragment ions. And then peaks were matched with the mzCloud (https://www.mzcloud.org/) and ChemSpider (http://www.chemspider.com/) database to obtain the accurate qualitative and relative quantitative results. PCA and PLS-DA were performed with MetaX software (BGI Tech, China) [17]. Variable Importance Projection (VIP) produced by PLS-DA and Fold Change (FC) were used to screen for differential metabolites with $\mathrm{P}$ value of T-test (VIP $>1, \mathrm{FC}>2.0$ or FC $<0.5$ and $\mathrm{P}<0.05$ ). Specificity and sensitivity of identified potential biomarkers were analyzed by ROC curves, and metabolic pathways involving potential biomarkers were topologically analyzed by the MetaboAnalyst 3.0 pathway analysis module (www.metaboanalyst.ca). LC-MS/MS assay was performed by Novogene Co., Ltd. (Beijing, China).

\subsection{Intestinal Flora Analysis}

Fecal DNA Extraction. Fecal genomic DNA was extracted using Qiagen QIAamp DNA Stool Mini Kit (Qiagen, Germany). DNA concentration and purity were monitored on $1 \%$ agarose gels. According to the concentration, DNA was diluted to $1 \mathrm{ng} / \mu \mathrm{L}$ using sterile water.

PCR Amplification and Product Purification. 16S rRNA genes of $16 \mathrm{~S}$ V4 regions were amplified using specific primer $(515 \mathrm{~F}$ $806 \mathrm{R}$ ) with the barcode. All PCR reactions were carried out in $30 \mu \mathrm{L}$ reactions with $15 \mu \mathrm{L}$ of Phusion ${ }^{\circledR}$ High-Fidelity PCR Master Mix (New England Biolabs, USA), $0.2 \mu \mathrm{M}$ of forward and reverse primers, and about $10 \mathrm{ng}$ template DNA. Thermal cycling consisted of initial denaturation at $98^{\circ} \mathrm{C}$ for $1 \mathrm{~min}$, followed by 30 cycles of denaturation at $98^{\circ} \mathrm{C}$ for $10 \mathrm{~s}$, annealing at $50^{\circ} \mathrm{C}$ for $30 \mathrm{~s}$, and elongation at $72^{\circ} \mathrm{C}$ for $30 \mathrm{~s}$ and finally $72^{\circ} \mathrm{C}$ for $5 \mathrm{~min}$. The same volume of $1 \times$ loading buffer (contained SYB green) was mixed with PCR products and electrophoresis was operated on 2\% agarose gel for detection. PCR products were mixed in equidensity ratios. Then, mixture PCR product was purified with GeneJET ${ }^{\mathrm{TM}} \mathrm{Gel}$ Extraction Kit (Thermo Scientific, USA).

Library Preparation and Sequencing Analysis. Sequencing libraries were generated using Ion Plus Fragment Library Kit 48 rxns (Thermo Scientific, USA) following manufacturer's recommendations. The library quality was assessed on the Qubit@2.0 Fluorometer (Thermo Scientific, USA). At last, the library was sequenced on an Ion S5TM XL platform (Thermo Scientific, USA) and $400 \mathrm{bp} / 600 \mathrm{bp}$ singleend reads were generated. The raw reads were spliced and filtered to obtain clean reads, and then all clean reads were clustered by Uparse software (Uparse v7.0.1001), sequences with $\geq 97 \%$ similarity were assigned to the same OTUs, and representative sequence for each OTU was screened for further species annotation through Silva Database (https://www.arb-silva.de/). Alpha diversity was analyzed by Observed Species, Shannon Index, and Chaol. Beta diversity was expressed as PCA. MetaStat and LEfSe methods were used to compare species with significant differences among groups. Spearman correlation coefficient was calculated to figure out the correlation between genus, species, and metabolites. 16S rDNA sequencing was performed by Novogene Co., Ltd. (Beijing, China).

\section{Results}

3.1. Blood Pressure and HE Staining. Systolic and diastolic blood pressure in HD group were not significantly different from those in $M$ group before treatment $(P>0.05)$. After 3 weeks of treatment, the diastolic blood pressure in $\mathrm{HD}$ group was significantly lower than that in $\mathrm{M}$ group $(\mathrm{P}<0.05)$. After 4 weeks of treatment, both systolic and diastolic blood pressure in HD group were lower than that in $\mathrm{M}$ group $(\mathrm{P}<$ 0.05) (Figure 1(a)). HE staining showed no significant inflammation and dysplasia in intestinal tissue in three groups (Figure 1(b)). Considering the chronic systemic low-grade inflammatory state in hypertension [3], pathological changes of intestine were not visually identified in HE staining.

3.2. Abundance and Diversity of Intestinal Flora. When the sequencing reads approached 80000 , the sequencing reached plateau stage suggesting full sequencing. The number of species was compared with W group $>$ HD group $>$ M group (Figure 2(a)). Chaol index was proportional to the abundance of intestinal flora and Shannon index was proportional to the diversity of intestinal flora. The abundance and diversity of intestinal flora in $\mathrm{M}$ group were all lower than those in $\mathrm{W}$ group, both of which recovered with different degrees after administration of HD (Figures 2(b) and 2(c)). Further PCA analysis was conducted based on Euclidean distances (Figure 2(d)), and the data suggested that the aggregation degree of samples in W group and HD group was better than that in M group, indicating that HD improved the disorder of intestinal flora in SHRs.

3.3. Screening of Intestinal Differential Flora. We compared the relative abundance of the three groups at the phylum, genus, and species levels. The top 5 phyla among the three groups were Firmicutes, Bacteroidetes, Verrucomicrobia, Actinobacteria, and Tenericutes (Figure 3(a)). In M group, the abundance of Firmicutes increased, the abundance of Bacteroidetes decreased, the ratio of Firmicutes to Bacteroidetes $(\mathrm{F} / \mathrm{B})$ increased, and the ratio of $\mathrm{F} / \mathrm{B}$ recovered after administration of HD (Figures 3(b), 3(c), and 3(d)). 

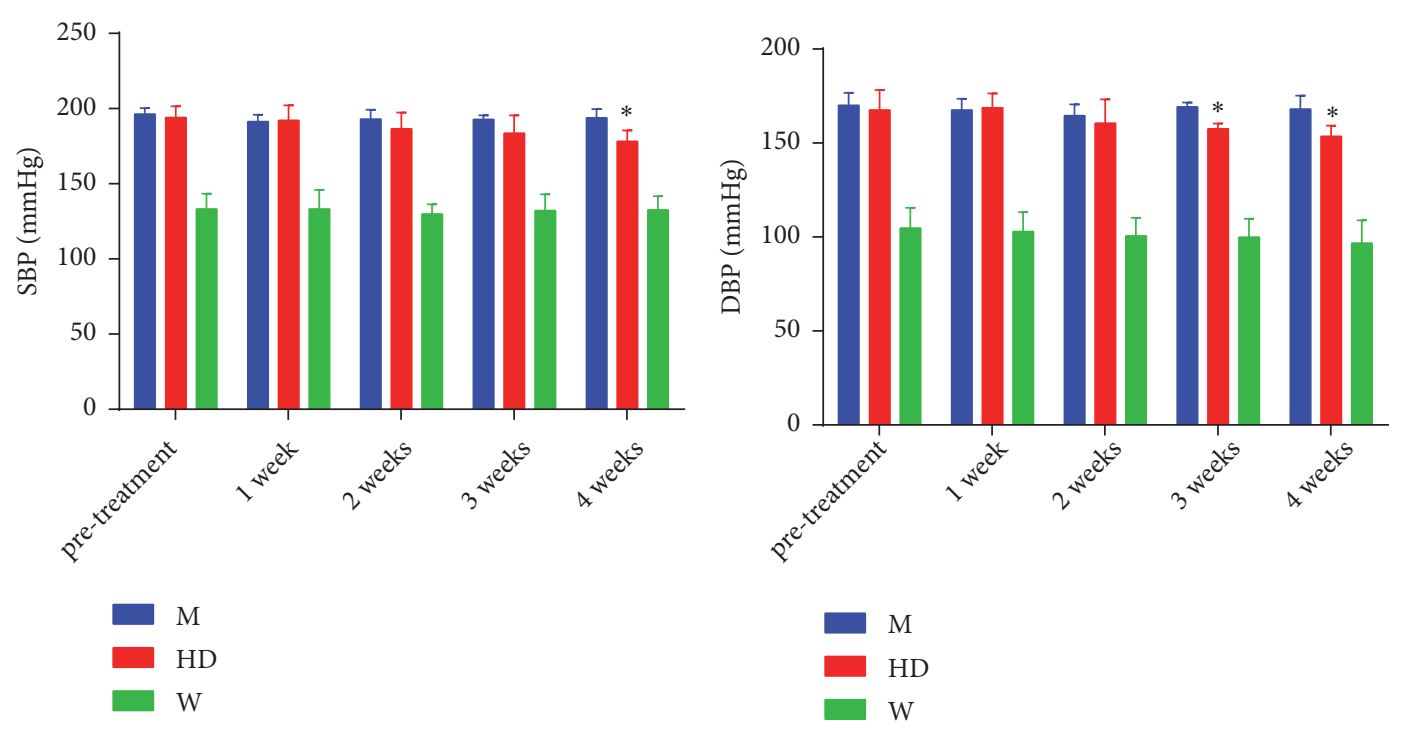

(a)
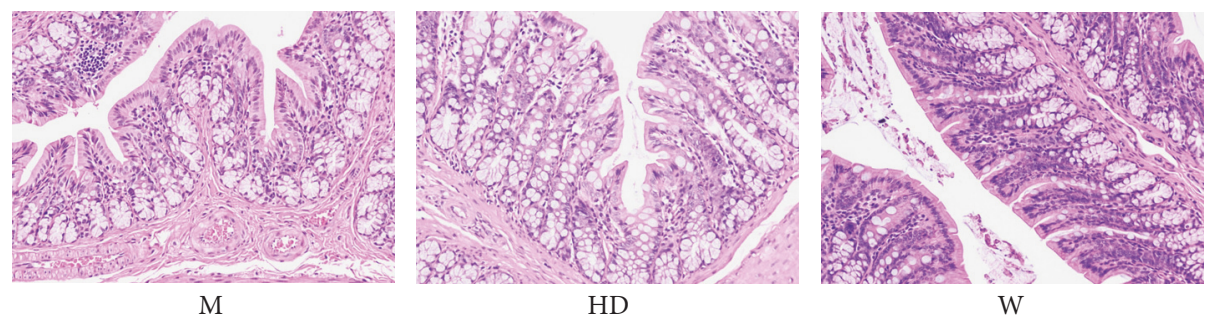

(b)

Figure 1: Blood pressure and HE staining. (a) Systolic and diastolic blood pressure changes in 4-week consecutive administration of HD. Results were compared by one-way ANOVA, $* \mathrm{P}<0.05$ compared with M group. (b) Histological changes of colon tissues in HE staining after 4-week consecutive administration of $\mathrm{HD}$ at 400 magnification.

We further used LEfSe (LDA score > 3.8) to analyze the statistically significant biomarkers among three groups. The dominant genuses and species of the $\mathrm{W}$ group were Lachnoclostridium, Faecalibaculum, and Lactobacillus reuteri, and the dominant genuses and species were Akkermansia, Akkermansia muciniphila, and Lactobacillus intestinalis in HD group; however, no dominant genus or species was found in M group (Figure 4(a)). The M group was further compared with the HD group and the $\mathrm{W}$ group to find the dominant genus and species. Compared with the HD group, the abundance of rumen bacterium NK4A214 was increased, and the abundance of Akkermansia, Akkermansia muciniphila, and Lactobacillus intestinalis was decreased in $\mathrm{M}$ group (Figure 4(b)). Compared with the $\mathrm{W}$ group, the abundance of Clostridium sp. MC 40 was increased, and the abundance of Lachnoclostridium, Faecalibaculum, and Lactobacillus reuteri was decreased in M group (Figure 4(c)).

3.4. Metabolic Pattern Recognition and Data Analysis. We analyzed the serum metabolism to validate efficacy of $\mathrm{HD}$. PCA analysis of the total samples showed that the QC samples were clustered, suggesting that the experimental data were well controlled. The metabolic patterns of $\mathrm{W}$ group were significantly different from those of HD group and M group (Figures 5(a) and 5(b)). The PCA and PLSDA analysis (Figures 5(c), 5(d), and 5(e)) were further performed in the $\mathrm{HD}$ group and the M group, and the metabolic patterns of the two groups were also significantly different. Compared with the M group, R2Y and Q2Y, representing the explanatory and predictive ability of PLSDA model, were close to 1 in W group and $\mathrm{HD}$ group, suggesting that the explanatory and predictive value of PLSDA model was high. The data demonstrated there were significant differences between WKY rats and SHRs in serum endogenous metabolites, and the serum metabolism of SHRs also changed significantly after administration of HD.

3.5. Identification of Potential Biomarkers. We screened differential metabolites by VIP $>1$, FC $>2.0$ or FC $<0.5$, and $\mathrm{P}$ value $<0.05$ (Figures $6(\mathrm{a})$ and $6(\mathrm{~b})$ ). Ultimately, 16 potential biomarkers were identified that may be associated with lowering blood pressure (Table 1). Details of the 16 metabolites were presented in the supplementary materials (Table S). Seven metabolites increased and 9 metabolites decreased in $\mathrm{M}$ group. 16 metabolites tended to be of normal level after intervention with HD. Receiver operating characteristic (ROC) curves were used to analyze the predictive ability of 16 potential biomarkers. By comparison with $M$ 


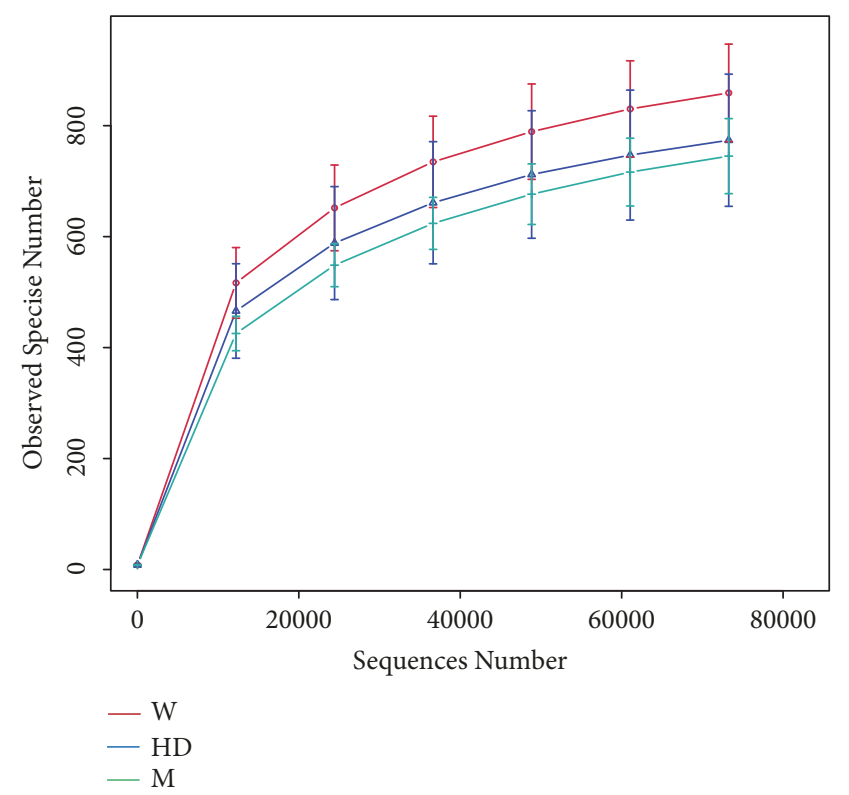

(a)

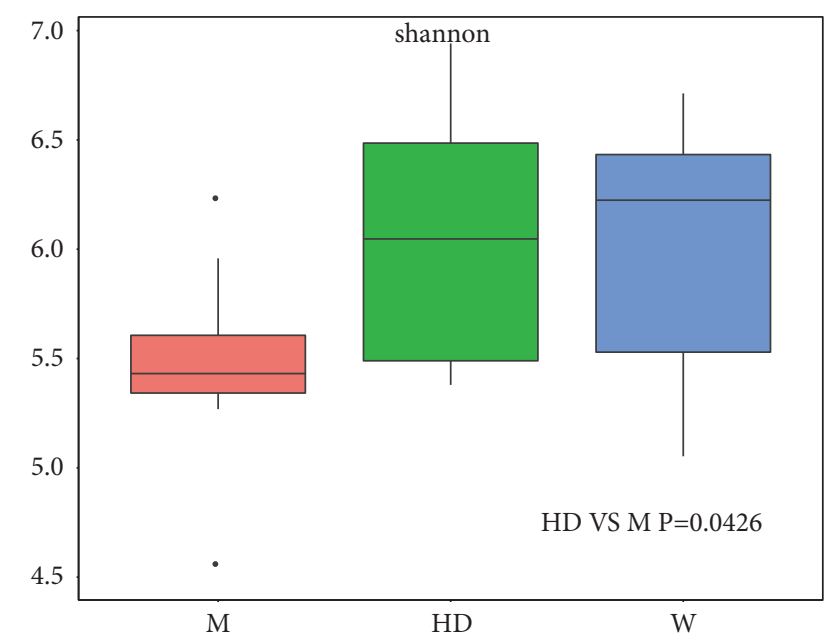

(c)

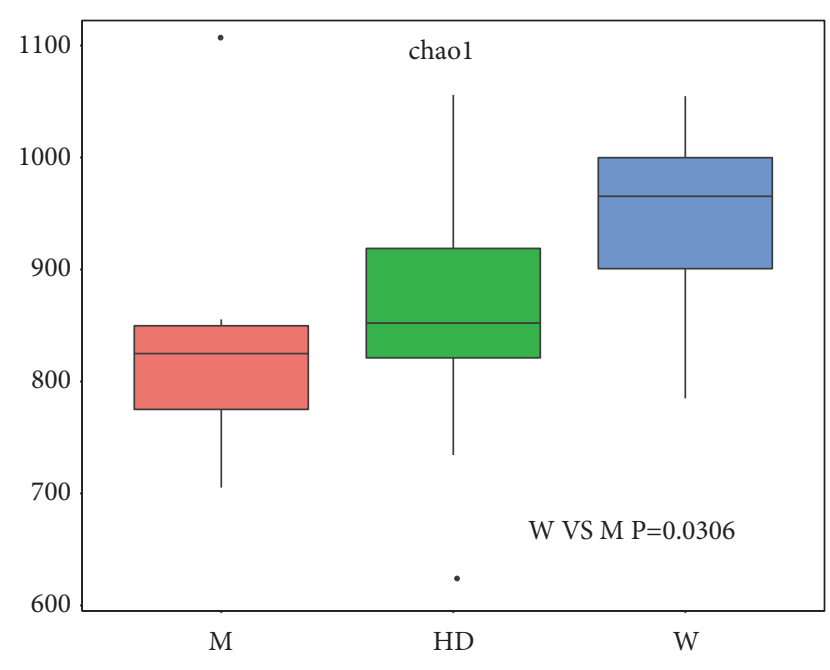

(b)

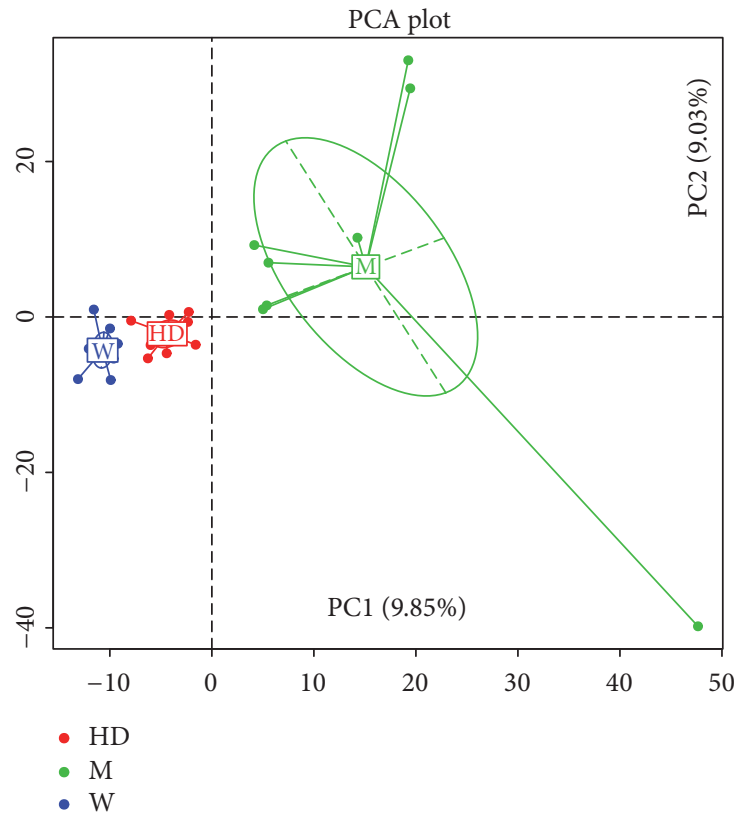

(d)

Figure 2: Abundance and diversity of intestinal flora. (a) Rarefaction curves of three groups for species number after 80000 random sequences number. The curve in each group was near smooth when the sequencing data were great enough with few new species undetected. ((b) and (c)) Box plot of difference among groups of Chao 1 index (used to assess abundance) and Shannon index (used to assess diversity). P values were from Wilcoxon rank sum test. (d) Principal Component Analysis (PCA) of three groups based on Euclidean distances. The percentage of PC1 and PC2 represented the contribution value of the first and second principal components to the sample difference.

group, all of them demonstrated high prediction accuracy, especially leukotriene B4, testosterone, prostaglandin E2, gamma-aminobutyric acid, arachidonic acid, L-glutathione (reduced), cortisone, and 5-hydroxyindoleacetic acid (Figure 7). Hierarchical clustering analysis of all differential metabolites including 16 potential biomarkers showed that the metabolic patterns and metabolic function of WKY rats and SHRs were different. The metabolic function of serum endogenous metabolites in SHRs changed significantly after the HD intervention (Figures 6(c) and 6(d)). To explore the metabolic function of the 16 potential biomarkers, we ran the pathway analysis module with Metaboanalys software. The results showed that arachidonic acid metabolism, tryptophan metabolism, glutathione metabolism, and steroid hormone 


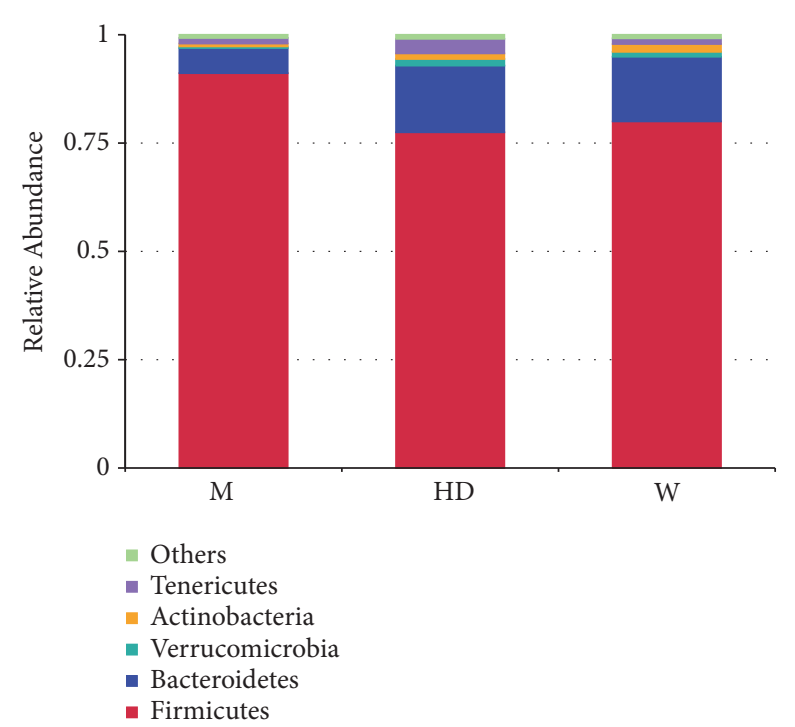

(a)

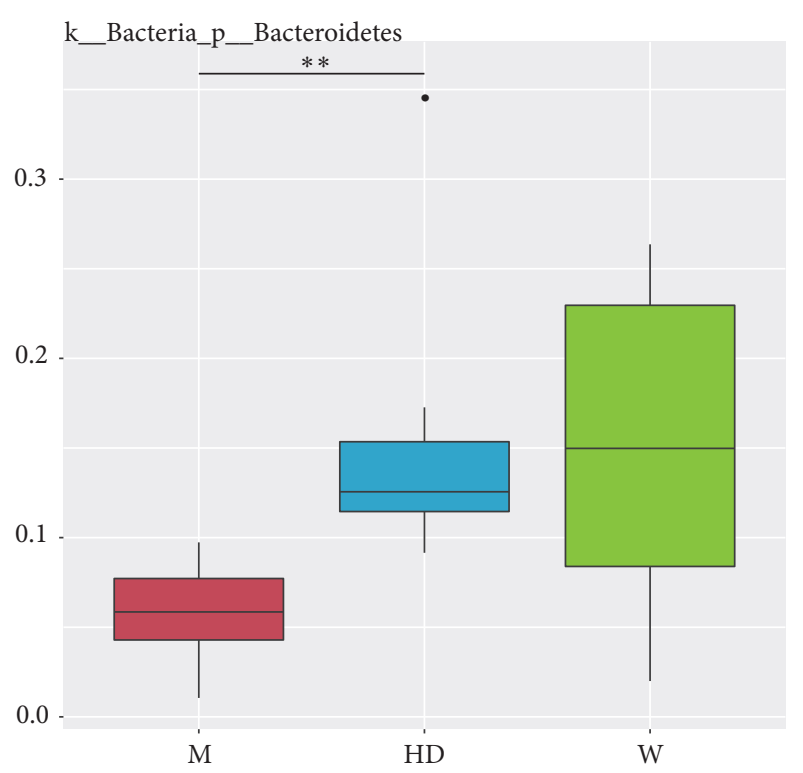

(c)

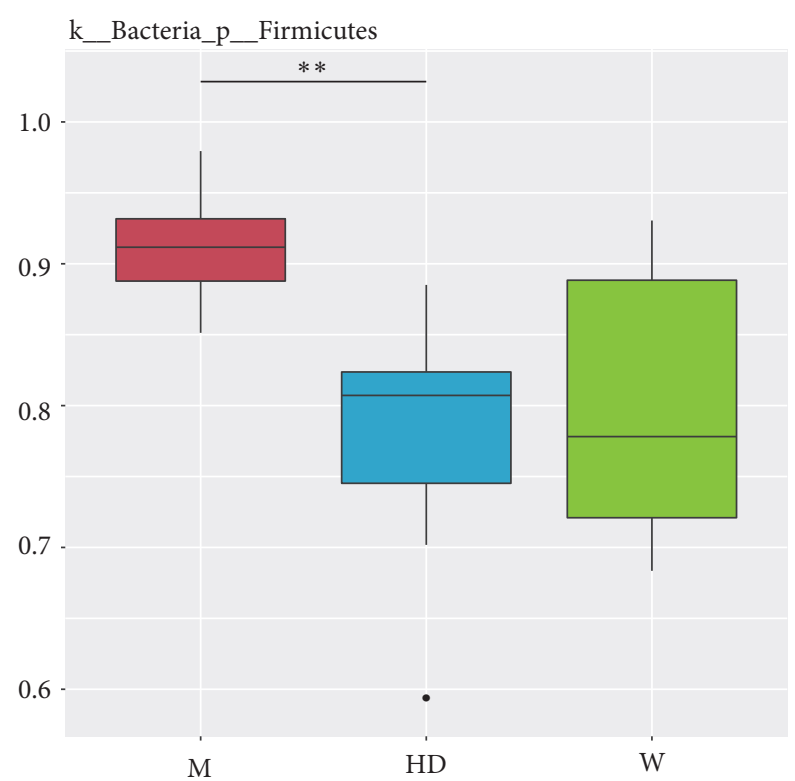

(b)

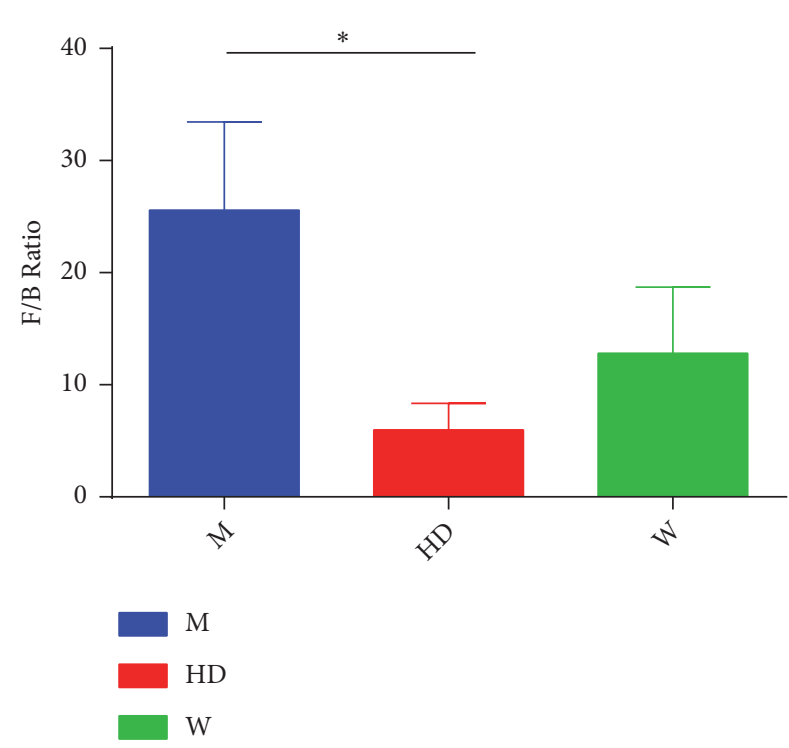

(d)

Figure 3: Changes of flora at the level of phylum. (a) The top 5 phylum of three groups. ((b) and (c)) Box plot of difference among three groups of Firmicutes and Bacteroidetes. The $\mathrm{p}$ value was obtained by the permutation test between groups and the $\mathrm{p}$ value was corrected by the Benjamini and Hochberg False Discovery Rate (FDR) method to obtain the q value, $* * \mathrm{q}<0.01$. (d) The ratio of Firmicutes to Bacteroidetes $(\mathrm{F} / \mathrm{B})$ in three groups. Results were compared by one-way ANOVA, $* \mathrm{P}<0.05$.

biosynthesis were the top 4 enrichment and topological pathways (Table 2, Figure 8).

3.6. Correlation between Potential Biomarkers and Differential Flora. Considering intestinal flora can regulate the metabolic function of the host, we compared the correlation of 8 significantly different genuses and species with 16 potential biomarkers using Spearman statistical method. Akkermansia and Akkermansia muciniphila were highly negatively correlated with indoleacetic acid and testosterone, while Lactobacillus intestinalis was highly positively correlated with 5-hydroxyindoleacetic acid and beta-estradiol and highly negatively correlated with indoleacetic acid and prostaglandin E2. Lachnoclostridium had a high positive correlation with L-kynurenine and 4-pyridinic acid, and a high negative correlation with arachidonic acid, leukotriene B4, and testosterone, while Lactobacillus reuteri was highly negatively correlated with leukotriene B4 and testosterone. Clostridium sp. MC 40 showed a high negative correlation with L-kynurenine and 4-pyridinic acid, and a high positive 
TABLE 1: 16 potential biomarkers among the three groups.

\begin{tabular}{|c|c|c|c|c|c|}
\hline Identification & Formula & Molecular Weight & $\begin{array}{c}\mathrm{RT} \\
(\mathrm{min}) \\
\end{array}$ & $\begin{array}{c}\text { Change Trend } \\
\left(\mathrm{HD}: \mathrm{M}^{\mathrm{a}} / \mathrm{W}: \mathrm{M}^{\mathrm{b}}\right)\end{array}$ & Pathway \\
\hline Taurocholic acid & $\mathrm{C}_{26} \mathrm{H}_{45} \mathrm{NO}_{7} \mathrm{~S}$ & 515.2900 & 14.3 & $\uparrow / \uparrow$ & $\begin{array}{c}\text { Primary bile acid } \\
\text { biosynthesis }\end{array}$ \\
\hline $\begin{array}{l}\text { 5-Hydroxyindoleacetic } \\
\text { acid }\end{array}$ & $\mathrm{C}_{10} \mathrm{H}_{9} \mathrm{NO}_{3}$ & 191.0580 & 8.36 & $\uparrow / \uparrow$ & $\begin{array}{l}\text { Tryptophan } \\
\text { metabolism }\end{array}$ \\
\hline Indoleacetic acid & $\mathrm{C}_{10} \mathrm{H}_{9} \mathrm{NO}_{2}$ & 175.0631 & 9.46 & $\downarrow / \downarrow$ & $\begin{array}{l}\text { Tryptophan } \\
\text { metabolism }\end{array}$ \\
\hline 4-Pyridoxic acid & $\mathrm{C}_{8} \mathrm{H}_{9} \mathrm{NO}_{4}$ & 183.0530 & 5.75 & $\uparrow / \uparrow$ & $\begin{array}{l}\text { Vitamin B6 } \\
\text { metabolism }\end{array}$ \\
\hline L-Kynurenine & $\mathrm{C}_{10} \mathrm{H}_{12} \mathrm{~N}_{2} \mathrm{O}_{3}$ & 208.0748 & 3.75 & $\uparrow / \uparrow$ & $\begin{array}{l}\text { Tryptophan } \\
\text { metabolism }\end{array}$ \\
\hline Adrenic acid & $\mathrm{C}_{22} \mathrm{H}_{36} \mathrm{O}_{2}$ & 332.2709 & 15.51 & $\downarrow / \downarrow$ & $\begin{array}{c}\text { Biosynthesis of } \\
\text { unsaturated fatty } \\
\text { acids }\end{array}$ \\
\hline Prostaglandin E2 & $\mathrm{C}_{20} \mathrm{H}_{32} \mathrm{O}_{5}$ & 352.2247 & 10.63 & $\downarrow / \downarrow$ & $\begin{array}{c}\text { Arachidonic acid } \\
\text { metabolism }\end{array}$ \\
\hline Leukotriene B4 & $\mathrm{C}_{20} \mathrm{H}_{32} \mathrm{O}_{4}$ & 336.2296 & 12.19 & $\downarrow / \downarrow$ & $\begin{array}{c}\text { Arachidonic acid } \\
\text { metabolism }\end{array}$ \\
\hline $\begin{array}{l}\text { Gamma-Aminobutyric } \\
\text { acid }\end{array}$ & $\mathrm{C}_{4} \mathrm{H}_{9} \mathrm{NO}_{2}$ & 103.0632 & 1.31 & $\uparrow / \uparrow$ & $\begin{array}{l}\text { beta-Alanine } \\
\text { metabolism }\end{array}$ \\
\hline Testosterone & $\mathrm{C}_{19} \mathrm{H}_{28} \mathrm{O}_{2}$ & 288.2088 & 13.85 & $\downarrow / \downarrow$ & $\begin{array}{c}\text { Steroid hormone } \\
\text { biosynthesis }\end{array}$ \\
\hline Sphinganine 1-phosphate & $\mathrm{C}_{18} \mathrm{H}_{40} \mathrm{NO}_{5} \mathrm{P}$ & 381.2641 & 14.59 & $\uparrow / \uparrow$ & $\begin{array}{l}\text { Sphingolipid } \\
\text { metabolism }\end{array}$ \\
\hline Arachidonic acid & $\mathrm{C}_{20} \mathrm{H}_{32} \mathrm{O}_{2}$ & 304.2399 & 13.62 & $\downarrow / \downarrow$ & $\begin{array}{l}\text { Arachidonic acid } \\
\text { metabolism }\end{array}$ \\
\hline Glycine & $\mathrm{C}_{2} \mathrm{H}_{5} \mathrm{NO}_{2}$ & 75.0320 & 6.34 & $\uparrow / \uparrow$ & $\begin{array}{l}\text { Glutathione } \\
\text { metabolism }\end{array}$ \\
\hline Cortisone & $\mathrm{C}_{21} \mathrm{H}_{28} \mathrm{O}_{5}$ & 360.1930 & 11.22 & $\downarrow / \downarrow$ & $\begin{array}{c}\text { Steroid hormone } \\
\text { biosynthesis }\end{array}$ \\
\hline Beta-Estradiol & $\mathrm{C}_{18} \mathrm{H}_{24} \mathrm{O}_{2}$ & 272.1774 & 11.83 & $\uparrow / \uparrow$ & $\begin{array}{c}\text { Steroid hormone } \\
\text { biosynthesis }\end{array}$ \\
\hline L-Glutathione (reduced) & $\mathrm{C}_{10} \mathrm{H}_{17} \mathrm{~N}_{3} \mathrm{O}_{6} \mathrm{~S}$ & 307.0833 & 1.50 & $\uparrow / \uparrow$ & $\begin{array}{l}\text { Glutathione } \\
\text { metabolism }\end{array}$ \\
\hline
\end{tabular}

Note. ${ }^{\mathrm{a}}$ Trends of HD group compared with M group. ${ }^{\mathrm{b}}$ Trends of W group compared with M group. $\uparrow:$ up-regulated. $\downarrow$ : down-regulated.

TABLE 2: Top 10 metabolic pathways of potential biomarkers.

\begin{tabular}{lccccccc}
\hline Pathway name & Total & Hits & $\mathrm{p}$ & Log(p) & Holm p & FDR & Impact \\
\hline Arachidonic acid metabolism & 36 & 3 & 0.0069239 & 4.9728 & 0.56084 & 0.40424 & 0.36753 \\
Tryptophan metabolism & 41 & 3 & 0.0099812 & 4.6071 & 0.79849 & 0.40424 & 0.10622 \\
Glutathione metabolism & 26 & 2 & 0.033842 & 3.3861 & 1 & 0.72468 & 0.36642 \\
Steroid hormone biosynthesis & 70 & 3 & 0.041817 & 3.1745 & 1 & 0.72468 & 0.07078 \\
Cyanoamino acid metabolism & 6 & 1 & 0.066665 & 2.7081 & 1 & 0.72468 \\
Biosynthesis of unsaturated fatty acids & 42 & 2 & 0.080649 & 2.5176 & 1 & 0.72468 \\
Taurine and hypotaurine metabolism & 8 & 1 & 0.087945 & 2.431 & 1 & 0.72468 \\
Primary bile acid biosynthesis & 46 & 2 & 0.094426 & 2.3599 & 1 & 0.72468 & 0.05952 \\
Methane metabolism & 9 & 1 & 0.098413 & 2.3186 & 1 & 0.72468 & 0 \\
Vitamin B6 metabolism & 9 & 1 & 0.098413 & 2.3186 & 1 & 0.72468 \\
\hline
\end{tabular}

Note. Total: the number of all metabolites in this pathway; Hits: The number of differential metabolites hitting this pathway; Raw p: P value obtained by enrichment analysis; Holm P: P value corrected by Holm-Bonferroni method; Impact: Impact factor from topological analysis. 

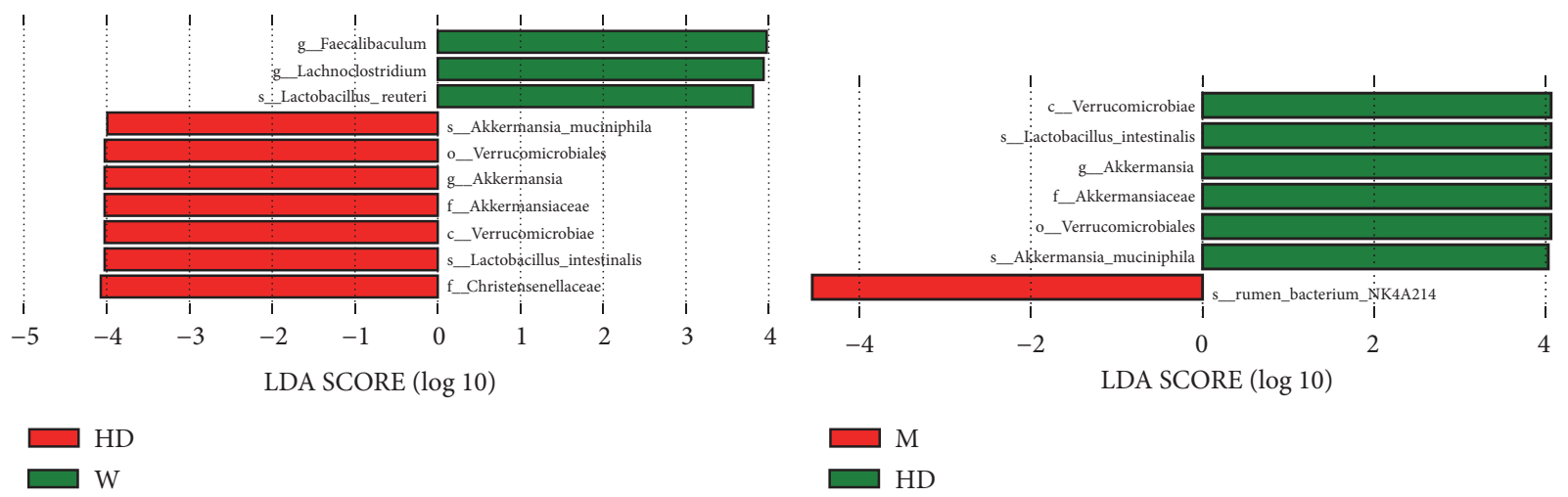

(a)

(b)

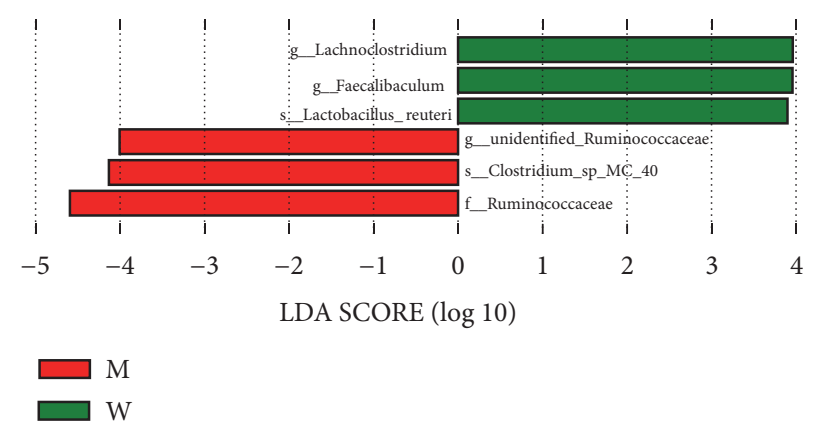

(c)

FIGURE 4: Screening of differential genus and species. A LEfSe analysis along with linear discriminate analysis (LDA) was applied to identify genus and species in three groups. Discriminative biomarkers with an LDA score $>3.8$; if one group was missing, it means there were no significant biomarkers in this group. (a) M group vs HD group vs W group (M group missing). (b) M group vs HD group. (c) M group vs W group.

correlation with indoleacetic acid, prostaglandin E2, and testosterone (Table 3).

\section{Discussion}

Both our results of the intestinal flora and serum metabolism indicated that $\mathrm{HD}$ administration demonstrated protective effects on SHRs. Correlation analysis indicated that the intestinal flora might concentrate on regulating metabolites in certain metabolic pathways, thereby reducing blood pressure.

Firmicutes and Bacteroidetes account for $90 \%$ or more in human intestinal flora. The ratio of Firmicutes to Bacteroidetes $(\mathrm{F} / \mathrm{B})$ and the richness, diversity, and evenness are important indicators reflecting the disorder of intestinal flora [1-3]. Our results suggested that the dominant phylum in three groups were similar to the human. The richness and diversity decreased and the F/B ratio of SHRs increased compared to those of WKY rats, which was consistent with previous studies in intestinal flora of SHRs and prehypertension and hypertension patients $[4,6]$. According to the recovered richness and diversity and decreased $\mathrm{F} / \mathrm{B}$ ratio after administration of $\mathrm{HD}$, we proposed that HD ameliorated the disorder of the structure and composition in SHRs' intestinal flora to a certain extent.
We further screened for differential flora that might restore intestinal flora disorder and lower blood pressure. The abundance of Akkermansia, Akkermansia muciniphila, and Lactobacillus intestinalis was increased after administration of HD. They were functionally similar to Lachnoclostridium, Faecalibaculum, and Lactobacillus reuteri, which had high abundance in WKY rats. Lactobacillus intestinalis has been verified at low level in hyperlipidemic rats and its abundance was inversely correlated with fat content and body weight [18], as well as the increased inflammatory response in rats with spinal cord injury [19]. Lactobacillus reuteri reduced chronic periodontitis [20] and improved the antioxidant and immune function of piglets [21]. In addition, Lactobacillus intestinalis and Lactobacillus reuteri all belong to lactobacillus producing lactic acid. Wilck et al. reported that high-salt diet decreased the level of lactobacillus and increased blood pressure [22]. As a bacteria producing butyric acid, Akkermansia muciniphila has been found with less abundance in obese and diabetic mice and patients, which improved hyperlipidemia and insulin resistance, reduced the level of circulating inflammatory factors, reduced endotoxemia, and improved intestinal barrier function [23, 24]. Lachnoclostridium as a butyrate-producing bacterium also improved obesity and insulin resistance $[25,26]$. Faecalibaculum was a newly isolated genus from mice in recent years and its main metabolic 


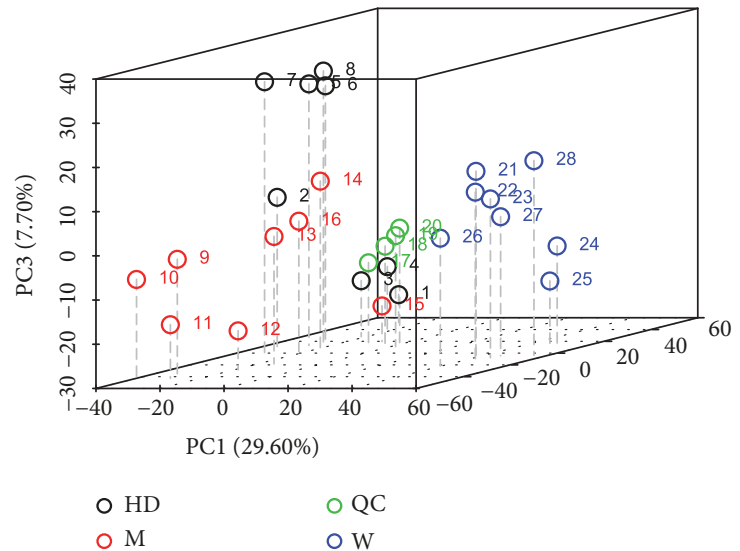

(a)

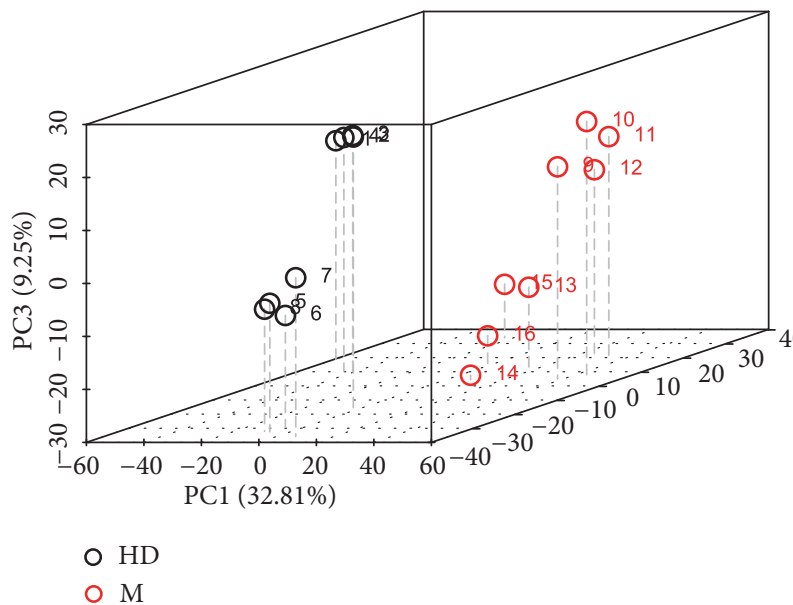

(c)

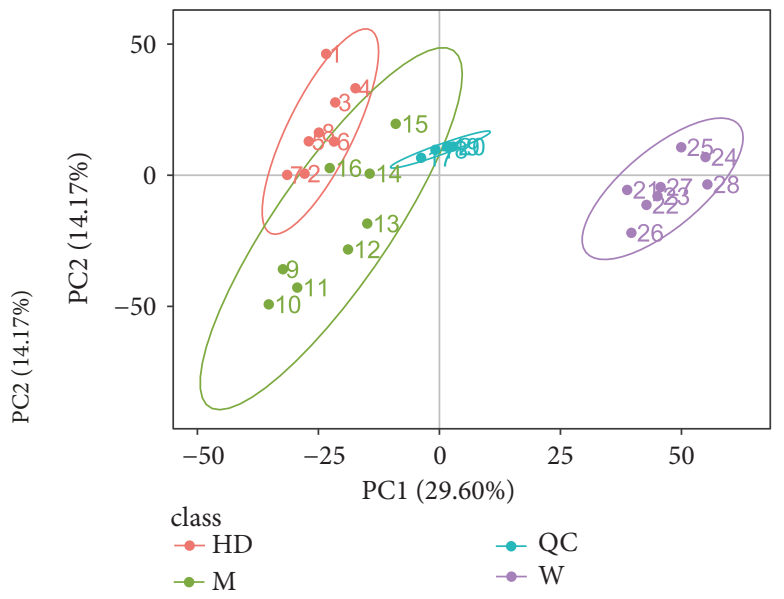

(b)

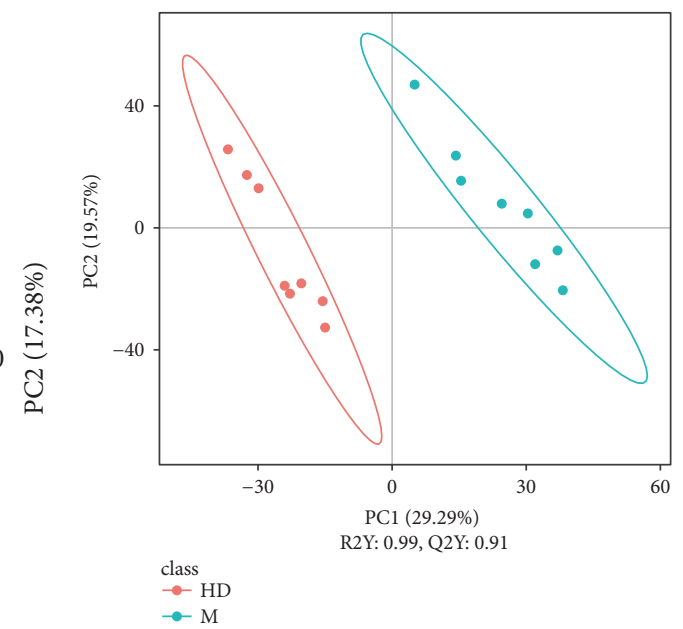

(d)

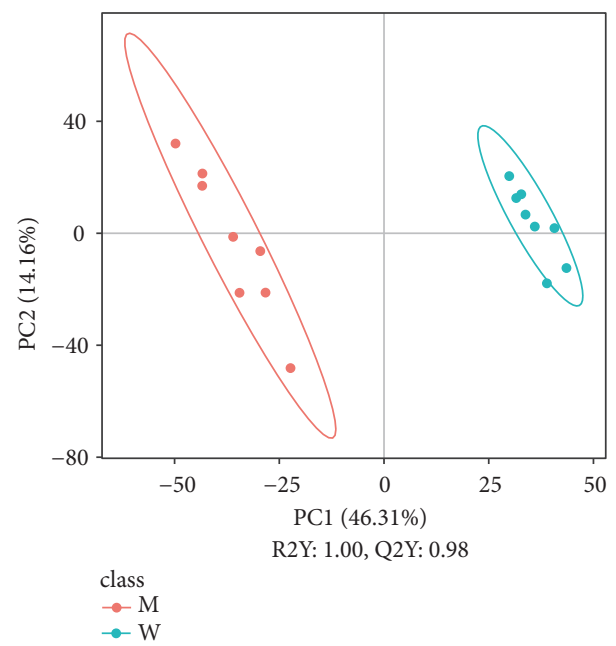

(e)

FIGURE 5: Metabolic pattern recognition and data analysis. ((a) and (b)) PCA score plot 3D and 2D of three groups and QC samples. PC1, first principle component score; PC2, second principle component score; PC3, third principle component score. The more aggregated the QC samples were, the higher the quality of experimental data was. Ellipses represented 95\% confidence intervals. (c) PCA score plot 3D (group HD vs M). ((d) and (e)) PLS-DA score plot 2D (group HD vs M; group W vs M). R2Y represented the interpretation rate of the second principal component of the model and Q2Y represented the prediction rate of the model. 

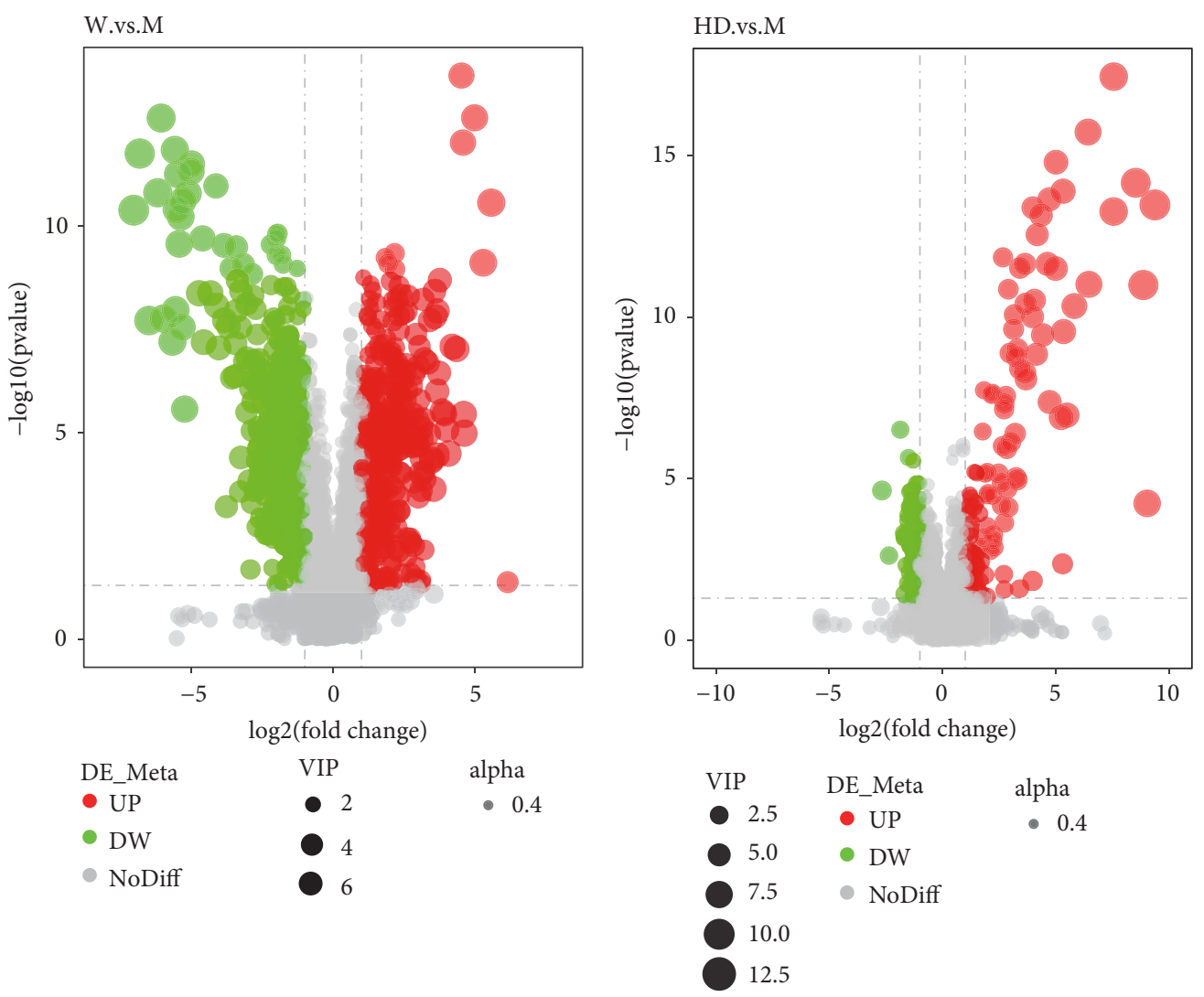

(a)

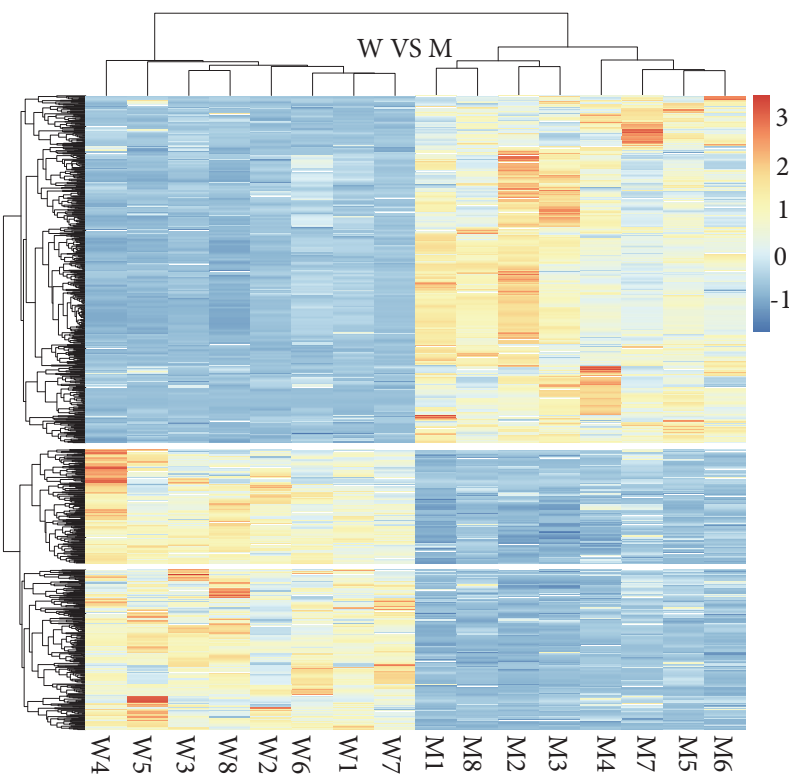

(c) (b)

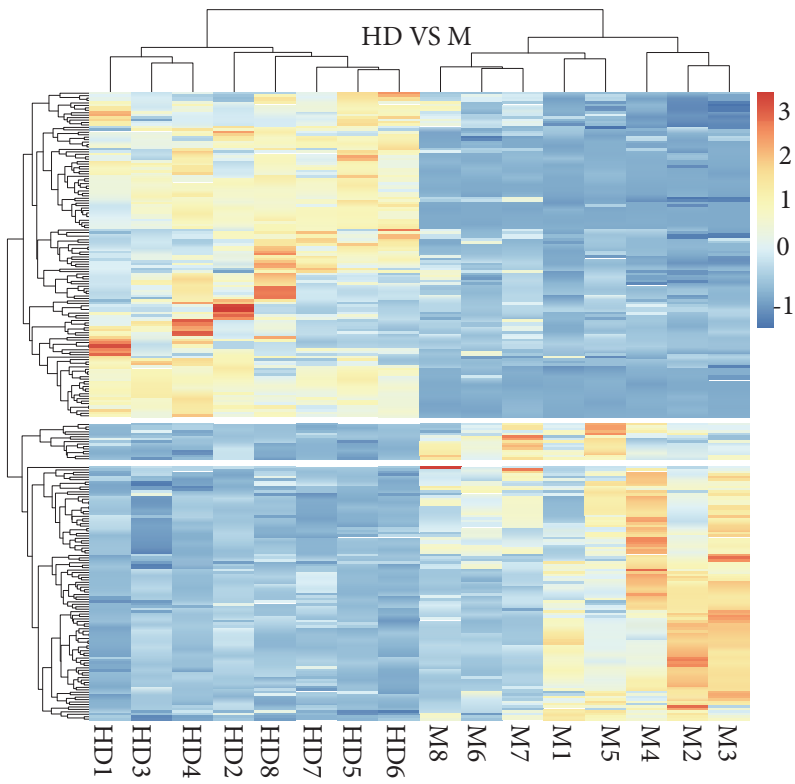

(d)

Figure 6: Identification of potential biomarkers. ((a) and (b)) Volcano Plot of differential metabolites (group W vs M; group HD vs M). The size of the dot represented the VIP value and alpha represented the transparency of the dot. Black represented metabolites that were not significantly different, red represented upregulated metabolites, and green represented downregulated metabolites. ((c) and (d)) Hierarchical clustering analysis of differential metabolites (group W vs M; group HD vs M). The clustering of the samples was longitudinal; the clustering of the metabolites was horizontal; the shorter the clustering branches, the more similar the color, and the higher the similarity. 


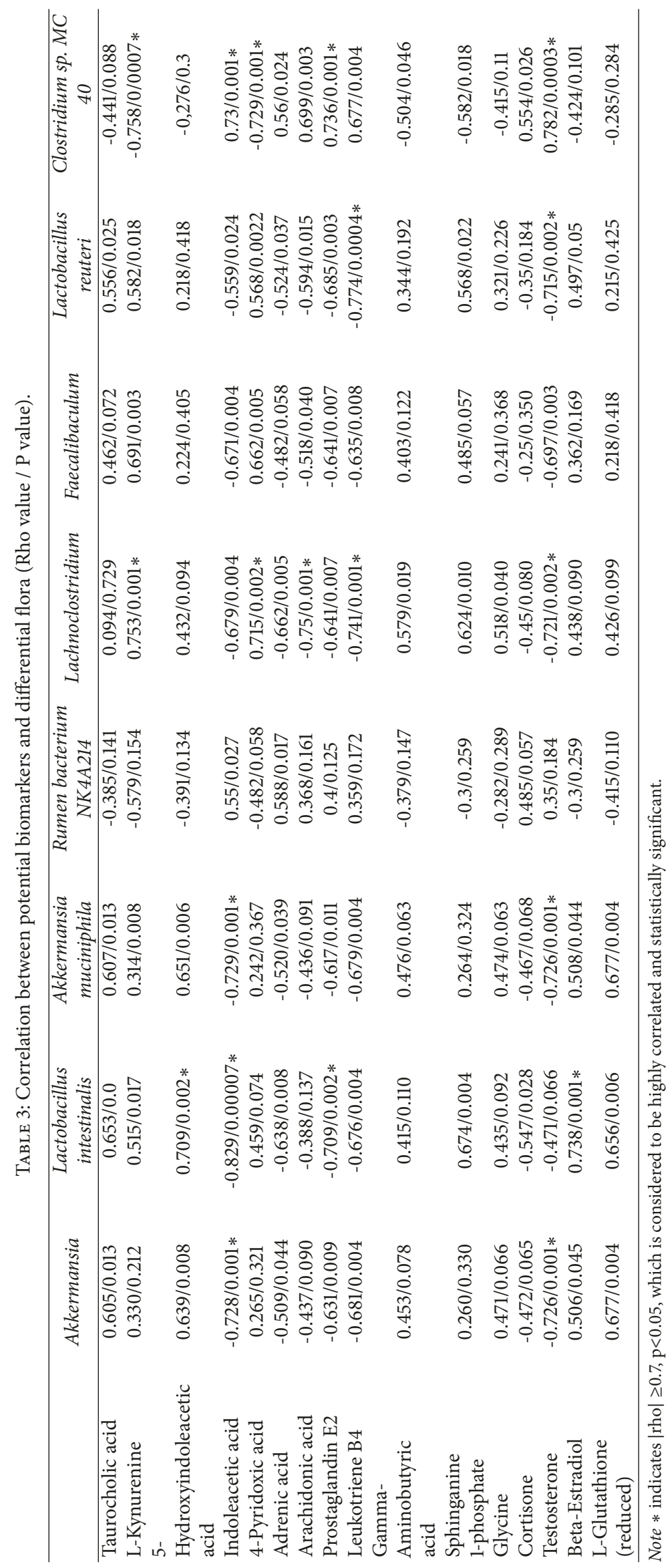



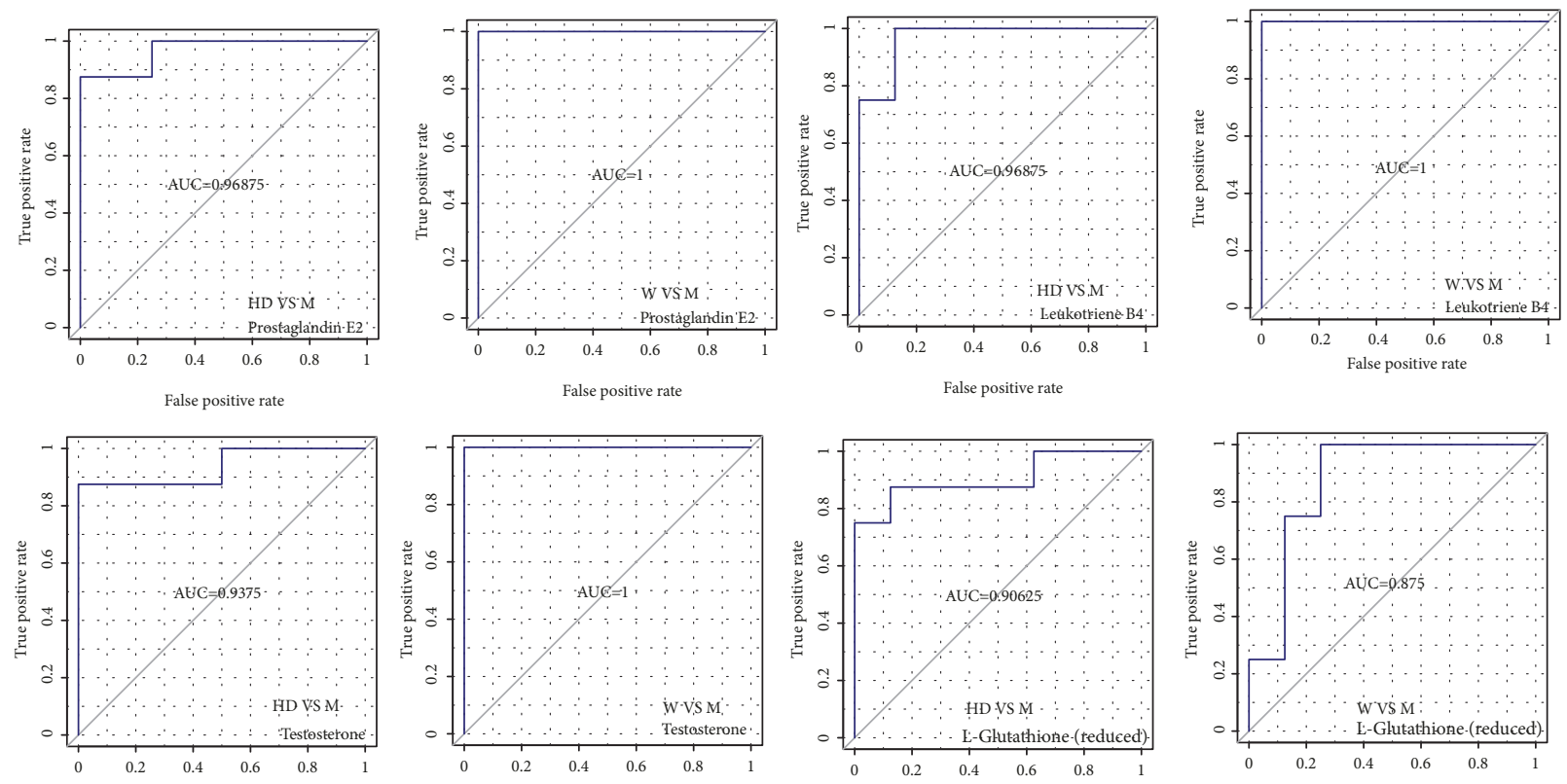

False positive rate

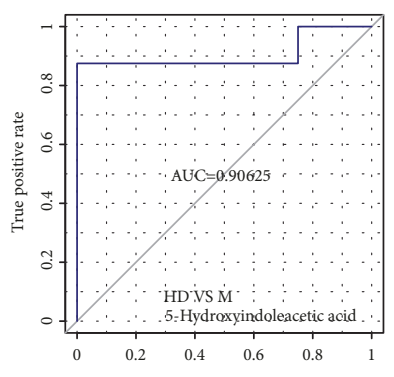

False positive rate
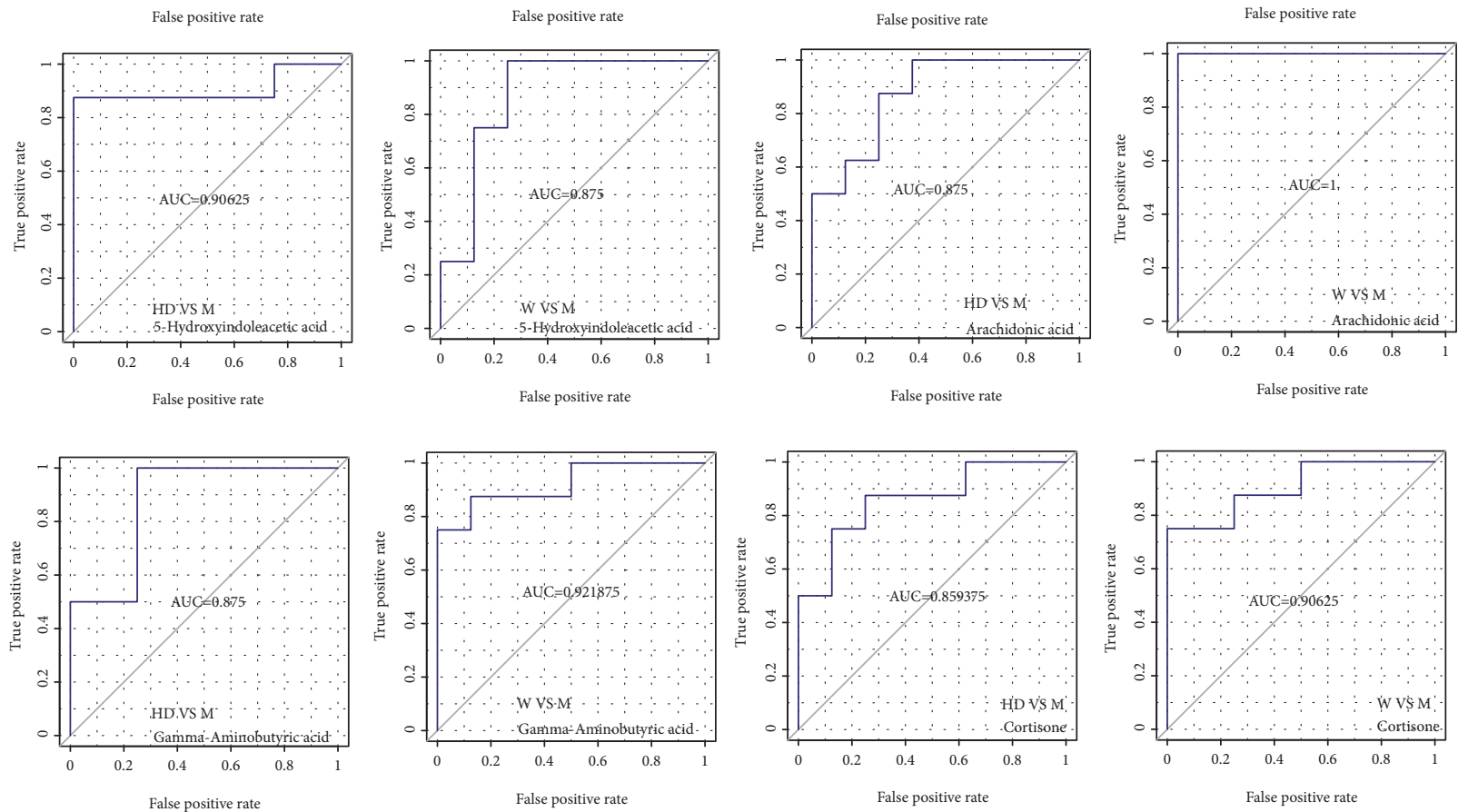

FIgURE 7: Receiver operating characteristic (ROC) curves analysis of potential biomarkers (group W vs M; group HD vs M). The area under the ROC curve was called Area Under Curve (AUC), which was used to assess the sensitivity and specificity of biomarkers for predicting events. We regarded AUC $>0.85$ as the bottom line of very high prediction accuracy.

end product was lactic acid [27]. Butyric acid, as the main component of short-chain fatty acids, regulated inflammation and immunity and improved intestinal barrier function [28]. Besides, the increase of butyric acid and lactic acid in the body promoted the abundance of each other's producing bacteria, thus forming a positive feedback loop and increasing the protective effect [29]. Our results identified the notion that the increased rumen bacterium NK4A214 and Clostridium sp. MC 40 in SHRs belong to unidentified Ruminococcaceae, which was not reported before and should be paid attention to. Our results suggested that Akkermansia, Akkermansia muciniphila, and Lactobacillus intestinalis might regulate blood pressure by regulating factors related to elevated blood pressure such as inflammation, blood lipids, and blood sugar. Besides, certain genus and species of Ruminococcaceae might be associated with elevated blood pressure.

Based on the fact that some of the final products of intestinal flora fermentation can enter the blood and exert 


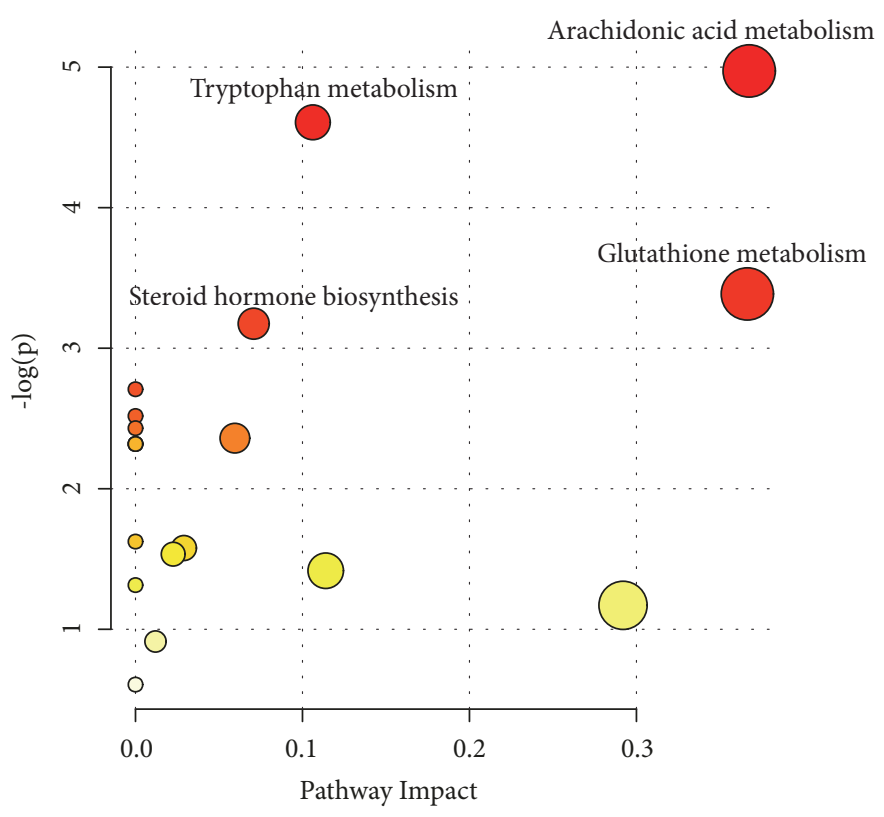

FIGURE 8: Metabolic pathway analysis of potential biomarkers. The color and size of circles were based on P value and pathway impact value, respectively. The larger the circle, the larger the impact factor; the darker the color, the smaller the $\mathrm{P}$ value, and the more significant the enrichment.

an important effect on the physiology of the host, we further studied the changes of serum metabolism of SHRs. 16 metabolites were screened out to be related to lowering blood pressure, all of which had very high accuracy of prediction, especially leukotriene B4, testosterone, prostaglandin E2, gamma-aminobutyric acid, arachidonic acid, L-glutathione (reduced), cortisone, and 5-hydroxyindoleacetic acid. We further explored the antihypertensive mechanism through the metabolic pathways involving 16 metabolites, which were mainly enriched in arachidonic acid metabolism, tryptophan metabolism, steroid hormones biosynthesis, and glutathione metabolism.

4.1. Arachidonic Acid Metabolism. The content of arachidonic acid (AA), prostaglandin E2 (PGE2), and leukotriene B4 (LTB4) in the arachidonic acid metabolism was all decreased after the administration of HD. AA was a substrate synthesizing a variety of proinflammatory, procoagulant, and vasoconstrictive substances and participating in the pathological process of hypertension [30], which was significantly positively correlated with the prevalence of hypertension and cardiovascular risk [31, 32]. PGE2 binds to its G protein-coupled receptors (GPCR) EP1 and EP3, leading to constricted blood vessels, platelet aggregation, inflammatory reactions, and increased blood pressure [33-35], which was involved in promoting salt-sensitive hypertension [36]. LTB4 is produced by AA via the 5-lipoxygenase (5-LOX)/leukotriene A4 (LTA4)/LTA4 hydrolase (LTA4H) pathway and is widely involved in inflammatory responses [33]. Relevant studies have found that LTB4 injection in SHRs' solitary bundle nucleus (NTS) caused elevated blood pressure, and the blocking of LTB4 receptor 1 (BLT1) significantly decreased blood pressure [37, 38]. HD might achieve the antihypertensive effect through anti-inflammatory, antiplatelet aggregation and vasodilatation by decreasing the level of the three metabolites.

4.2. Tryptophan Metabolism. The content of L-kynurenine (L-kyn) and 5-hydroxyindoleacetic acid (5-HIAA) in the tryptophan metabolism increased after administration of $\mathrm{HD}$, while the content of indoleacetic acid (IAA) decreased. Tryptophan is an essential amino acid of the human body, which is decomposed via serotonin (5-HT) and kyn. IAA is a metabolite of the kyn pathway, and 5-HIAA is a metabolite of the 5-HT pathway. Repeated release of 5-HT contracted blood vessels, promoted platelet aggregation, and raised blood pressure [39]. 5-HT serotonin transporter (SERT) could reuptake 5-HT and degrade it into 5-HIAA in intestinal epithelial cells or nerve cells, alleviating the enterotoxicity and neurotoxicity of free 5-HT [40]. Indoleamine 2,3 dioxygenase (IDO) metabolized tryptophan to kyn and thereby decreased the blood pressure of inflammatory mice, while inhibition of IDO activity reduced the generation of kyn and then rebounded blood pressure [41]. Studies has found that kylinase (KYNU) mRNA levels were elevated in SHRs' brainstem, since KYNU catalyzed Kyn as 3-hydroxyanthronic acid (3HAA), and 3-HAA promoted AA to produce prostaglandin $\mathrm{E}$ and leukotriene under the action of Cyclooxygenase 2 (COX2) and 5-LOX, both of which were closely related to the increase of blood pressure [42, 43]. HD might restore the disturbance of L-kyn, 5-HIAA, and IAA by regulating the activities of SERT, IDO, and KYNU in tryptophan metabolism, which probably interact with arachidonic acid in the process of antihypertension. 
4.3. Steroid Hormone Biosynthesis. Steroid hormones include sex hormones and corticosteroids. After administration of $\mathrm{HD}$, the contents of testosterone and cortisone in SHRs' serum decreased, while the contents of beta-estradiol increased. Testosterone is the main component of androgen and estradiol is the most active component of estrogen. Both testosterone supplementation and reduction of 17 betaestradiol activated the RAAS system in vivo to increase blood pressure $[44,45]$. Cortisone, as a glucocorticoid, promoted the reabsorption of water and sodium in the kidneys and increased blood pressure after being transformed into the active hydrocortisone [46]. Affecting the sex hormones and corticosteroid levels of SHRs may be involved in antihypertensive effect of HD.

4.4. Glutathione and Other Metabolism. We also observed that the level of glutathione (reduced), glycine, taurocholic acid, 4-pyridoxic acid, gamma-aminobutyric acid, and sphingosine 1-phosphate increased and adrenic acid decreased after administration of HD. Glycine is one of the components of reduced glutathione, which enhanced the activity of superoxide dismutase (SOD) and glutathione peroxidase (GSH-Px), scavenged oxygen free radicals, and improved the capability against oxidative stress $[47,48]$. Taurocholic acid, an important product of bile acid metabolism, reduced liver lipid accumulation [49]. 4-pyridic acid was a metabolite of vitamin B6, and the low level of vitamin B6 was closely related to inflammatory status and cardiovascular mortality [50]. Gamma-aminobutyric acid, as an inhibitory neurotransmitter, has been found to be decreased in salt-sensitive hypertensive rats, and its increase decreased SHRs' blood pressure [51, 52]. Sphingosine 1-phosphate was catalyzed by sphingosine kinase (SPK) through the S1P receptor. The activation of SPK/S1P signaling pathway activated eNOS and protected myocardial cells from acute ischemia-reperfusion $[53,54]$. As an omega- 6 polyunsaturated fatty acid, adrenic acid acted as an inflammatory mediator as a ligand of immune receptor [55]. These changes of metabolites regulated by $\mathrm{HD}$ may participate in the antihypertensive process through antiinflammatory and antioxidative stress effects.

We further analyzed the correlation between genius, species, and metabolites. Akkermansia, Akkermansia muciniphila, and Lactobacillus intestinalis were highly correlated with testosterone, beta-estradiol, indoleacetic acid, 5-hydroxyindoleacetic acid, and prostaglandin E2, which were enriched in steroid hormone biosynthesis, tryptophan metabolism, and arachidonic acid metabolism. Previous studies have found that excessive androgen exposure during perinatal period reduced the levels of Akkermansia and Lactobacillus in intestinal and increased blood pressure [56]. Our study also found elevated levels of androgen and decreased estrogen levels in SHRs' serum, and the levels of Akkermansia, Akkermansia muciniphila, and Lactobacillus intestines in SHRs' intestinal decreased, while HD improved the disorder. Testosterone, L-kynurenine, arachidonic acid, leukotriene B4, and 4-pyridinic acid, which were highly associated with the dominant Lachnoclostridium and Lactobacillus reuteri in WKY rats, were enriched in steroid hormone biosynthesis, arachidonic acid metabolism, tryptophan metabolism, and vitamin B6 metabolism. Lachnoclostridium was also highly correlated with vitamin B6 metabolism and tryptophan metabolism in mice with colitis [57], similar to our study in WKY rats. Coincidentally, testosterone, L-kynurenine, indoleacetic acid, prostaglandin E2, and 4-pyridinic acid, which were highly correlated with the dominant Clostridium sp. MC 40 in SHRs, were also enriched in steroid hormone biosynthesis, tryptophan metabolism, arachidonic acid metabolism, and vitamin B6 metabolism. In our study, the metabolites and their enriched metabolic pathways, which were closely related to the three dominant geniuses and species, were highly concentrated. We therefore speculated that the intestinal flora may be the target of HD administration, thus to regulate steroid hormone biosynthesis, arachidonic acid metabolism, tryptophan metabolism, and vitamin B6 metabolism.

\section{Conclusion}

In conclusion, the present study indicated that the combination of Astragalus membranaceus and Salvia miltiorrhiza demonstrated good antihypertensive effect, which may be driven by the protective intestinal flora and beneficial metabolites. This study provided new insights into the antihypertensive mechanism of Astragalus membranaceus and Salvia miltiorrhiza.

\section{Data Availability}

The data used to support the findings of this study are included within the article. If additional support data are needed, they are available from the corresponding author or first author upon request. First author's mailbox is hancong_kidney@hotmail.com. Corresponding author's mailbox is lweidw@163.com.

\section{Conflicts of Interest}

The authors declare that they have no competing interests.

\section{Authors' Contributions}

Cong Han performed the experiment and drafted the manuscript. Yue-hua Jiang performed the experiment and revised the manuscript. Yao Liu carried out data collection and data analysis of 16s rRNA and LC-MS/MS. Zhen-qiang Qi was responsible for animal care and behaviour observation. Wei Li designed the experiment and made critical revision of the manuscript. All authors read and approved the final manuscript.

\section{Acknowledgments}

This work was funded by National Natural Science Foundation of China (no. 81673812) and Key Research \& Development Program of Shandong Province (no. 2018GSF119017). 


\section{Supplementary Materials}

Details of the 16 metabolites were presented in the supplementary materials (Table S). (Supplementary Materials)

\section{References}

[1] H. A. Jama, D. M. Kaye, and F. Z. Marques, "The gut microbiota and blood pressure in experimental models," Current Opinion in Nephrology and Hypertension, vol. 28, no. 2, pp. 97-104, 2019.

[2] A. Aguilar, "Hypertension: Microbiota under pressure," Nature Reviews Nephrology, vol. 13, no. 1, 3 pages, 2017.

[3] Y. Yu, G. Mao, J. Wang et al., "Gut dysbiosis is associated with the reduced exercise capacity of elderly patients with hypertension," Hypertension Research, vol. 41, no. 12, pp. 1036-1044, 2018.

[4] T. Yang, M. M. Santisteban, V. Rodriguez et al., "Gut dysbiosis is linked to hypertension," Hypertension, vol. 65, no. 6, pp. 13311340, 2015.

[5] S. Khalesi, J. Sun, N. Buys, and R. Jayasinghe, "Effect of probiotics on blood pressure: a systematic review and metaanalysis of randomized, controlled trials," Hypertension, vol. 64, no. 4, pp. 897-903, 2014.

[6] J. Li, F. Zhao, Y. Wang et al., "Gut microbiota dysbiosis contributes to the development of hypertension," Microbiome, vol. 5, no. 1, pp. 1-19, 2017.

[7] M. Kordalewska and M. J. Markuszewski, "Metabolomics in cardiovascular diseases," Journal of Pharmaceutical and Biomedical Analysis, vol. 113, pp. 121-136, 2015.

[8] Y. Chu, H. Jiang, J. Ju et al., "A metabolomic study using HPLCTOF/MS coupled with ingenuity pathway analysis: intervention effects of Rhizoma Alismatis on spontaneous hypertensive rats," Journal of Pharmaceutical and Biomedical Analysis, vol. 117, pp. 446-452, 2016.

[9] W. Yang, Y. Deng, H. Zhou et al., "Metabolic characteristics of Rhizoma Coptidis intervention in spontaneously hypertensive rats: Insights gained from metabolomics analysis of serum," Molecular Medicine Reports, vol. 16, no. 4, pp. 4301-4308, 2017.

[10] A. Liu, Y.-J. Chu, X. Wang et al., "Serum metabolomics study based on lc-ms and antihypertensive effect of uncaria on spontaneously hypertensive rats," Evidence-Based Complementary and Alternative Medicine, vol. 2018, Article ID 9281946, 11 pages, 2018.

[11] N. Zhang, X.-H. Wang, S.-L. Mao, and F. Zhao, "Astragaloside IV improves metabolic syndrome and endothelium dysfunction in fructose-fed rats," Molecules, vol. 16, no. 5, pp. 3896-3907, 2011.

[12] J. Zhang, S. J. An, J. Q. Fu et al., "Mixed aqueous extract of salvia miltiorrhiza reduces blood pressure through inhibition of vascular remodelling and oxidative stress in spontaneously hypertensive rats," Cellular Physiology and Biochemistry, vol. 40, no. 1-2, pp. 347-360, 2016.

[13] M. Ali, T. Khan, K. Fatima et al., "Selected hepatoprotective herbal medicines: Evidence from ethnomedicinal applications, animal models, and possible mechanism of actions," Phytotherapy Research, vol. 32, no. 2, pp. 199-215, 2018.

[14] J. Fu, Z. H. Wang, L. F. Huang et al., "Review of the botanical characteristics, phytochemistry, and pharmacology of Astragalus membranaceus (Huangqi)," Phytotherapy Research, vol. 28, no. 9, pp. 1275-1283, 2014.

[15] X.-H. Ma, Y. Ma, J.-F. Tang et al., "The biosynthetic pathways of tanshinones and phenolic acids in salvia miltiorrhiza," Molecules, vol. 20, no. 9, pp. 16235-16254, 2015.
[16] L. Qin, H.-L. Tan, Y.-G. Wang et al., "Astragalus membranaceus and salvia miltiorrhiza ameliorate lipopolysaccharide-induced acute lung injury in rats by regulating the toll-like receptor 4/nuclear factor-Kappa B signaling pathway," Evidence-Based Complementary and Alternative Medicine, vol. 2018, Article ID 3017571, 10 pages, 2018.

[17] B. Wen, Z. Mei, C. Zeng, and S. Liu, "metaX: a flexible and comprehensive software for processing metabolomics data," BMC Bioinformatics, vol. 18, no. 1, p. 183, 2017.

[18] V. Lecomte, N. O. Kaakoush, C. A. Maloney et al., "Changes in gut microbiota in rats fed a high fat diet correlate with obesityassociated metabolic parameters," PLoS ONE, vol. 10, no. 5, p. e0126931, 2015.

[19] G. O’Connor, E. Jeffrey, D. Madorma et al., "Investigation of microbiota alterations and intestinal inflammation post-spinal cord injury in rat model," Journal of Neurotrauma, vol. 35, no. 18, pp. 2159-2166, 2018.

[20] S. Ikram, N. Hassan, S. Baig, K. J. Borges, M. A. Raffat, and Z. Akram, "Effect of local probiotic (Lactobacillus reuteri) vs systemic antibiotic therapy as an adjunct tonon-surgical periodontal treatment in chronic periodontitis," Journal of Investigative and Clinical Dentistry, vol. 10, no. 2, Article ID e12393, 2019.

[21] J. Yang, C. Wang, L. Liu, and M. Zhang, "Lactobacillus reuteri KT260178 supplementationreduced morbidity of piglets through its targeted colonization, improvement of cecal microbiota profile, and immune functions," Probiotics and Antimicrobial Proteins, pp. 1-10, 2019.

[22] N. Wilck, M. G. Matus, S. M. Kearney et al., "Salt-responsive gut commensal modulates TH17 axis and disease," Nature, vol. 551, no. 7682, pp. 585-589, 2017.

[23] H. Plovier, A. Everard, C. Druart et al., "A purified membrane protein from Akkermansia muciniphila or the pasteurized bacterium improves metabolism in obese and diabetic mice," Nature Medicine, vol. 23, no. 1, pp. 107-113, 2017.

[24] M. C. Dao, A. Everard, J. Aron-Wisnewsky et al., “Akkermansia muciniphila and improved metabolic health during a dietary intervention in obesity: relationship with gut microbiome richness and ecology," Gut, vol. 65, no. 3, pp. 426-436, 2016.

[25] V. Jacquier, A. Nelson, M. Jlali, L. Rhayat, K. S. Brinch, and E. Devillard, "Bacillus subtilis 29784 induces a shift in broiler gut microbiome toward butyrate-producing bacteria and improves intestinal histomorphology and animal performance," Poultry Science, vol. 98, no. 6, pp. 2548-2554, 2019.

[26] P. Wang, D. Li, W. Ke, D. Liang, X. Hu, and F. Chen, "Resveratrol-induced gut microbiota reduces obesity in highfat diet-fed mice," International Journal of Obesity, 2019.

[27] D.-H. Chang, M.-S. Rhee, S. Ahn et al., "Faecalibaculum rodentium gen. nov., sp. nov., isolated from the faeces of a laboratory mouse," Antonie van Leeuwenhoek-Journal of Microbiology, vol. 108, no. 6, pp. 1309-1318, 2015.

[28] H. Ohira, W. Tsutsui, and Y. Fujioka, "Are short chain fatty acids in gut microbiota defensive players for inflammation and atherosclerosis?" Journal of Atherosclerosis and Thrombosis, vol. 24, no. 7, pp. 660-672, 2017.

[29] J. Ye, L. Lv, W. Wu et al., "Butyrate protects mice against methionine-choline-deficient diet-induced non-alcoholic steatohepatitis by improving gut barrier function, attenuating inflammation and reducing endotoxin levels," Frontiers in Microbiology, vol. 9, no. 1967, 2018. 
[30] U. N. Das, "Arachidonic acid in health and disease with focus on hypertension and diabetes mellitus: A review," Journal of Advanced Research, vol. 11, pp. 43-55, 2018.

[31] P. Tsou and C. Wu, "Sex-dimorphic association of plasma fatty acids with cardiovascular fitness in young and middle-aged general adults: subsamples from nhanes 2003-2004," Nutrients, vol. 10, no. 10, p. 1558, 2018.

[32] I. Tsukamoto and S. Sugawara, "Low levels of linoleic acid and $\alpha$-linolenic acid and high levels of arachidonic acid in plasma phospholipids are associated with hypertension," Biomedical Reports, vol. 8, no. 1, pp. 69-76, 2018.

[33] V. Capra, M. Bäck, S. S. Barbieri, M. Camera, E. Tremoli, and G. E. Rovati, "Eicosanoids and their drugs in cardiovascular diseases: focus on atherosclerosis and stroke," Medicinal Research Reviews, vol. 33, no. 2, pp. 364-438, 2013.

[34] W. S. Harris, D. Mozaffarian, E. Rimm et al., "Omega-6 fatty acids and risk for cardiovascular disease: a science advisory from the American Heart Association nutrition subcommittee of the council on nutrition, physical activity, and metabolism; council on cardiovascular nursing; and council on epidemiology and prevention," Circulation, vol. 119, no. 6, pp. 902-907, 2009.

[35] G. Yang and L. Chen, "An update of microsomal prostaglandin E Synthase-1 and $\mathrm{PGE}_{2}$ receptors in cardiovascular health and diseases," Oxidative Medicine and Cellular Longevity, vol. 2016, Article ID 5249086, 9 pages, 2016.

[36] M.-Z. Zhang, B. Yao, Y. Wang et al., "Inhibition of cyclooxygenase-2 in hematopoietic cells results in saltsensitive hypertension," The Journal of Clinical Investigation, vol. 125, no. 11, pp. 4281-4294, 2015.

[37] P. J. Marvar, E. B. Hendy, T. D. Cruise et al., “ Systemic leukotriene B 4 receptor antagonism lowers arterial blood pressure and improves autonomic function in the spontaneously hypertensive rat ," The Journal of Physiology, vol. 594, no. 20, pp. 5975-5989, 2016.

[38] H. Waki, E. B. Hendy, C. C. Hindmarch et al., "Excessive leukotriene B4 in nucleus tractus solitarii is prohypertensive in spontaneously hypertensive rats," Hypertension, vol. 61, no. 1, pp. 194-201, 2013.

[39] R. Amstein, N. Fetkovska, A. Pletscher, and F. R. Bühler, "Platelet deactivation by 5HT2-receptor blockade parallels the antihypertensive response to ketanserin," Journal of Hypertension, vol. 7, no. 4, pp. 255-260, 1989.

[40] M. D. Gershon, "5-Hydroxytryptamine (serotonin) in the gastrointestinal tract," Current Opinion in Endocrinology, Diabetes and Obesity, vol. 20, no. 1, pp. 14-21, 2013.

[41] Y. Wang, H. Liu, G. McKenzie et al., "Kynurenine is an endothelium-derived relaxing factor produced during inflammation," Nature Medicine, vol. 16, no. 3, pp. 279-285, 2010.

[42] K. Mizutani, K. Sugimoto, T. Okuda et al., "Kynureninase is a novel candidate gene for hypertension in spontaneously hypertensive rats," Hypertension Research, vol. 25, no. 1, pp. 135140, 2002.

[43] G. F. Oxenkrug, "Metabolic syndrome, age-associated neuroendocrine disorders, and dysregulation of tryptophan-kynurenine metabolism," Annals of the New York Academy of Sciences, vol. 1199, no. 1, pp. 1-14, 2010.

[44] C. Dalmasso, C. N. Patil, L. L. Yanes Cardozo, D. G. Romero, and R. O. Maranon, "Cardiovascular and metabolic consequences of testosterone supplements in young and old male spontaneously hypertensive rats: implications for testosterone supplements in men," Journal of the American Heart Association, vol. 6, no. 10, Article ID e007074, 2017.

[45] T. Imanishi, T. Hano, and I. Nishio, "Estrogen reduces angiotensin II-induced acceleration of senescence in endothelial progenitor cells," Hypertension Research, vol. 28, no. 3, pp. 263-271, 2005.

[46] M. S. Cooper and P. M. Stewart, "PM.11Beta-hydroxysteroid dehydrogenase type 1 and its role in the hypothalamuspituitary-adrenal axis,metabolic syndrome,and inflammation," The Journal of Clinical Endocrinology \& Metabolism, vol. 94, no. 12, pp. 4645-4654, 2009.

[47] N. Jiang, L. Lu, T. Wang, L. Zhang, W. Xin, and F. Fu, "Reduced glutathione attenuates liver injury induced by methyl parathion in rats," Toxicology Mechanisms and Methods, vol. 20, no. 2, pp. 69-74, 2010.

[48] C. Li, F. Jiang, Y. Li et al., "Rhynchophylla total alkaloid rescues autophagy, decreases oxidative stress and improves endothelial vasodilation in spontaneous hypertensive rats," Acta Pharmacologica Sinica, vol. 39, no. 3, pp. 345-356, 2018.

[49] R. Sun, N. Yang, B. Kong et al., "Orally administered berberine modulates hepatic lipid metabolism by altering microbial bile acid metabolism and the intestinal FXR signaling pathway," Molecular Pharmacology, vol. 91, no. 2, pp. 110-122, 2017.

[50] A. Ulvik, E. R. Pedersen, G. F. Svingen et al., "Vitamin B6 catabolism and long-term mortality risk in patients with coronary artery disease," American Journal of Clinical Nutrition, vol. 103, no. 6, pp. 1417-1425, 2016.

[51] K. Kawakami, K. Yamada, T. Yamada et al., "Antihypertensive effect of $\gamma$-aminobutyric acid-enriched brown rice on spontaneously hypertensive rats," Journal of Nutritional Science and Vitaminology, vol. 64, no. 1, pp. 56-62, 2018.

[52] M. Wang, Y. Kang, X. Li et al., "Central blockade of NLRP3 reduces blood pressure via regulating inflammation microenvironment and neurohormonal excitation in salt-induced prehypertensive rats," Journal of Neuroinflammation, vol. 15, no. 1, p. 95, 2018.

[53] S. Salomone and C. Waeber, "Selectivity and specificity of sphingosine-1-phosphate receptor ligands: Caveats and critical thinking in characterizing receptor-mediated effects," Frontiers in Pharmacology, vol. 2, pp. 9-14, 2011.

[54] H. Yu, X. Che, X. Xu et al., "Insulin protects apoptotic cardiomyocytes from hypoxia/reoxygenation injury through the sphingosine kinase/sphingosine 1-phosphate axis," PLoS ONE, vol. 8, no. 12, p. e80644, 2013.

[55] A. Marco-Ramell, S. Tulipani, M. Palau-Rodriguez et al., "Untargeted profiling of concordant/discordant phenotypes of high insulin resistance and obesity to predict the risk of developing diabetes," Journal of Proteome Research, vol. 17, no. 7, pp. 2307-2317, 2018.

[56] S. B. Sherman, N. Sarsour, M. Salehi et al., "Prenatal androgen exposure causes hypertension and gut microbiota dysbiosis," Gut Microbes, vol. 9, no. 5, pp. 400-421, 2018.

[57] S. Haange, N. Jehmlich, M. Hoffmann et al., "Disease development is accompanied by changes in bacterial protein abundance and functions in a refined model of dextran sulfate sodium (DSS)-induced colitis," Journal of Proteome Research, vol. 18, no. 4, pp. 1774-1786, 2019. 


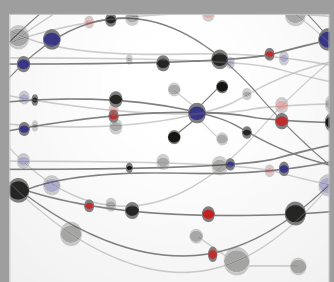

The Scientific World Journal
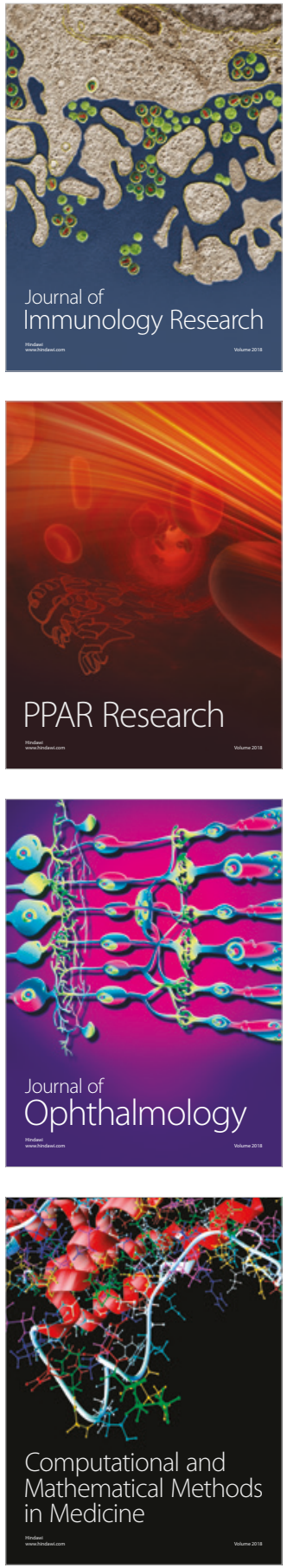

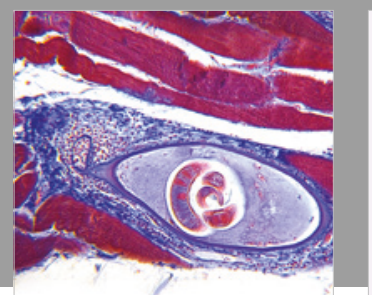

Gastroenterology Research and Practice

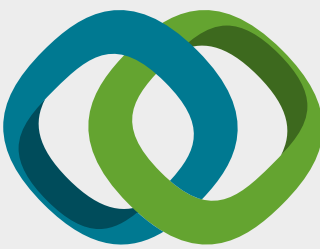

\section{Hindawi}

Submit your manuscripts at

www.hindawi.com
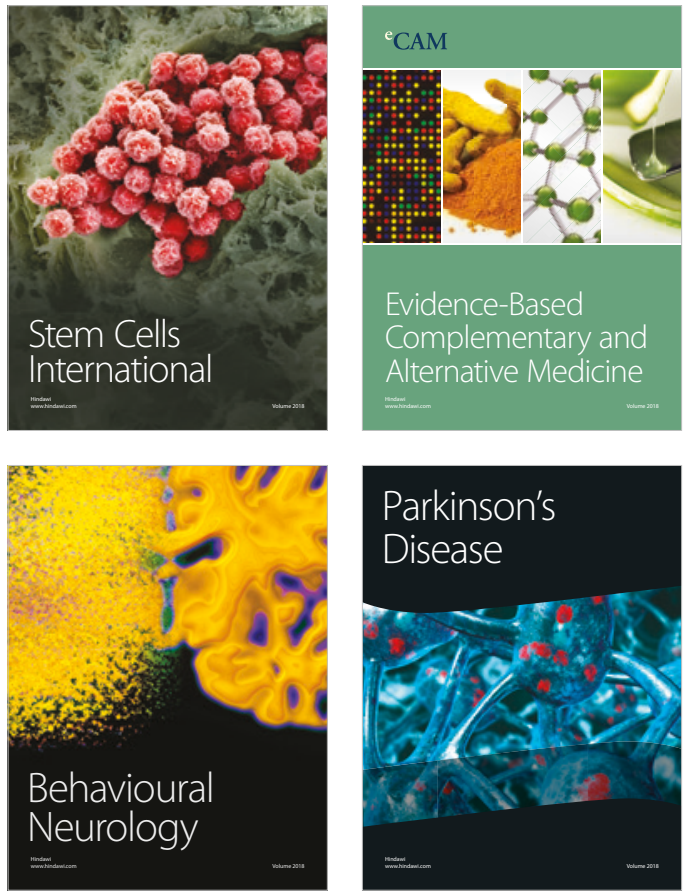

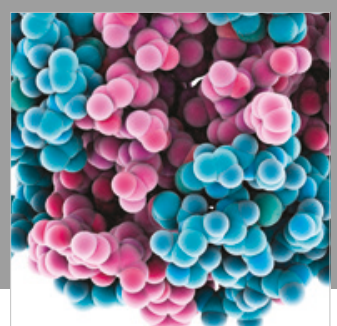

ournal of

Diabetes Research

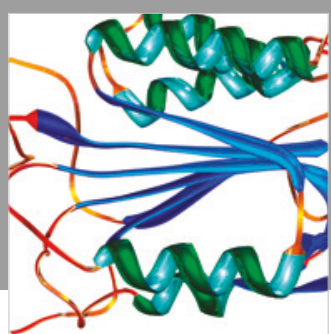

Disease Markers
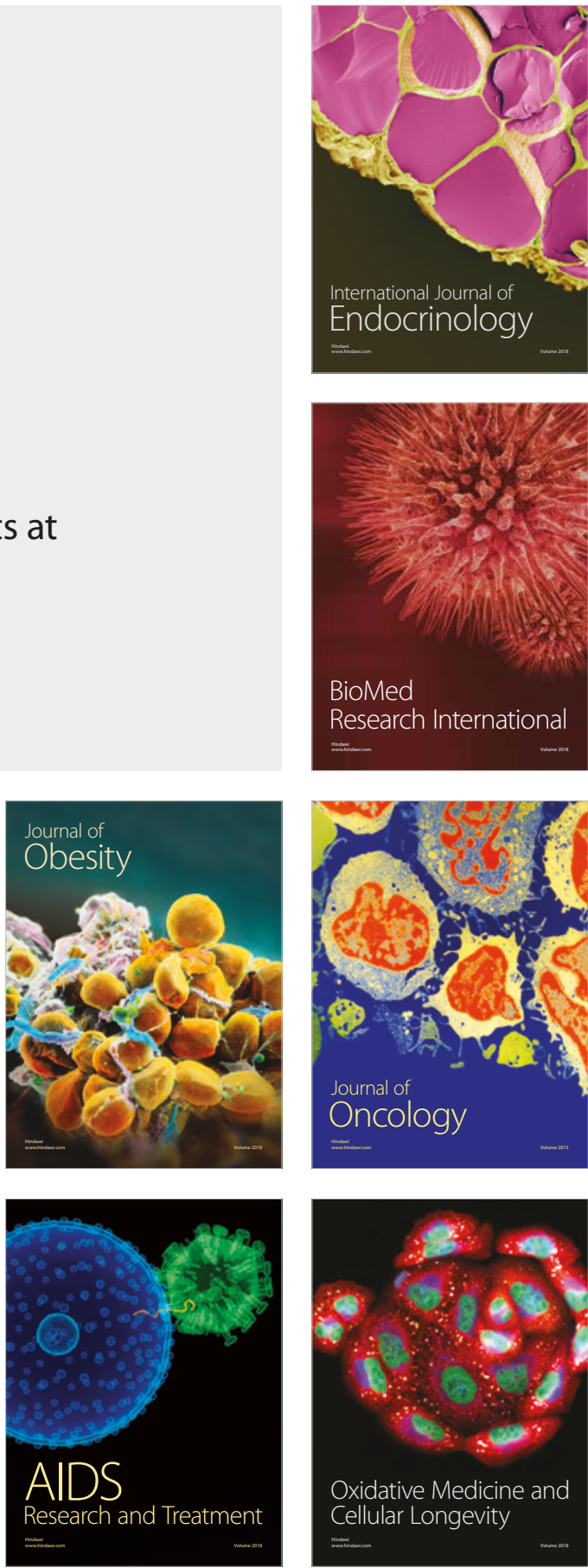